\title{
A branch-and-cut algorithm for the Time Window Assignment Vehicle Routing Problem
}

\author{
Kevin Dalmeijer* and Remy Spliet \\ Econometric Institute \\ Erasmus School of Economics \\ Erasmus University Rotterdam
}

October 19, 2016

EI2016-39

\begin{abstract}
This paper presents a branch-and-cut algorithm for the Time Window Assignment Vehicle Routing Problem (TWAVRP), the problem of assigning time windows for delivery before demand volume becomes known. A novel set of valid inequalities, the precedence inequalities, is introduced and multiple separation heuristics are presented. In our numerical experiments the branch-and-cut algorithm is 3.8 times faster when separating precedence inequalities. Furthermore, in our experiments, the branch-and-cut algorithm is 193.9 times faster than the best known algorithm in the literature. Finally, using our algorithm, instances of the TWAVRP are solved which are larger than the small scale instances previously presented in the literature.

Keywords: Vehicle Routing, Time Window Assignment, Precedence Inequalities. MSC codes: $90 B 06$ (Transportation), 90C11 (Mixed integer programming), 90C57 (Branch-and-cut).
\end{abstract}

*E-mail: dalmeijer@ese.eur.nl; Phone: +3110 408 9059; Address: PO Box 1738, 3000DR Rotterdam, The Netherlands; Corresponding author 


\section{Introduction}

The Time Window Assignment Vehicle Routing Problem (TWAVRP) is the problem of assigning time windows for delivery to clients in a distribution network when the demand volume of the clients is uncertain, such that the expected transport costs are minimized. First introduced by Spliet and Gabor (2014), the TWAVRP is inspired by distribution networks of retailers.

In a retail network, the clients are retail stores whom place orders on a regular basis. It is common that the time windows for delivery are fixed for a long period of time (e.g., a year). This is convenient for the retailers, as it allows them to ensure that enough personnel is available to process the delivery. Furthermore, it simplifies inventory control. Demand is uncertain and fluctuates per delivery. This results in orders of varying sizes, which become known shortly before the vehicles are dispatched.

To deal with demand uncertainty, the TWAVRP requires demand scenarios and their probability of occurrence to be known in advance. Note that if the number of scenarios is equal to one, the problem reduces to a Vehicle Routing Problem with Time Windows (VRPTW). Hence, the TWAVRP is NP-hard.

An increasing number of companies focus on achieving consistent service with their deliveries (Kovacs et al., 2014). Also in the scientific literature, we see the same trend towards consistency considerations in routing, as can be seen in the recent survey by Kovacs et al. (2014). In this survey, three main pillars of consistency are described: arrival time, person-oriented and delivery consistency, and the TWAVRP is categorized within the first. Our study adds to the limited amount of research done so far on exact methods for solving routing problems with consistency considerations.

Among the routing problems with consistency considerations, the TWAVRP is in particular closely related to two specific models. Firstly, the TWAVRP is similar to the Consistent Vehicle Routing Problem (ConVRP) introduced in Groër et al. (2009). The ConVRP does not only impose consistent arrival time but also requires the same driver to service the same customer. Another closely related model is the Vehicle Routing Problem with self-imposed time windows, as introduced by Jabali et al. (2015). In their paper, the authors assume demand to be given while travel times are uncertain.

The TWAVRP is a generalization of both the Capacitated Vehicle Routing Problem (CVRP) and the VRPTW, and hence similar solution methods can be used. In a recent survey, Baldacci et al. (2012) mention there are three formulations that have been most successful when used to solve the CVRP. Two of them make use of flow variables (Laporte et al. (1985), Baldacci et al. (2004)), while the third is a set partitioning formulation (Balinski and Quandt, 1964). For the VRPTW, a set partitioning formulation in a branch-price-and-cut algorithm is very successful (Desaulniers et al., 2008). To solve the TWAVRP, Spliet and Gabor (2014) also introduce a branch-price-and-cut algorithm based on a set partitioning formulation, which allows for instances with up to 25 clients and three demand scenarios to be solved to optimality within a one hour time limit. Similarly, Spliet and Desaulniers (2015) solve the DTWAVRP, the discrete time window variant of the TWAVRP.

In this paper, we present a new flow formulation for the TWAVRP that is of polynomial size in the number of clients and scenarios. This formulation is based on the MTZinequalities in Miller et al. (1960) and the 2-commodity flow formulation in Baldacci et al. (2004). Based on this formulation, we construct a branch-and-cut algorithm that 
is faster than the algorithm in Spliet and Gabor (2014). This new algorithm does not only allow for obtaining solutions faster, but also allows for solving larger instances of the TWAVRP, making it applicable to larger networks than previously possible.

One of the factors that contributes to the success of the branch-and-cut algorithm, is the introduction of a novel class of valid inequalities specifically designed for the TWAVRP: the precedence inequalities. We identify pairs of routes in different scenarios that cannot be selected simultaneously for any feasible time window assignment. Because time windows are not fixed in advance, identifying such pairs is a main challenge, which we address. We subsequently create valid inequalities using these pairs, similar to the valid inequalities designed by Ascheuer et al. (2000), which disallow infeasible paths for the Asymmetric Traveling Salesman Problem with Time Windows (ATSPTW). We show that separating precedence inequalities is co-NP-hard and present several separation heuristics to find violated inequalities.

The remainder of this paper is structured as follows: in Section 2, we present the formulation that is used in our branch-and-cut algorithm. In Section 3 we present the branchand-cut framework. Section 4 is dedicated to the precedence inequalities and heuristics for separating them. Our numerical experiments and their results are presented in Section 5. In the final section, we write our conclusions and present some directions for further research.

\section{Problem definition}

In this section, we first formally introduce the TWAVRP. Then, we present a mixed integer linear program to solve the problem.

Consider a set of $n$ clients $V^{\prime}=\{1,2, \ldots, n\}$. Furthermore, location 0 represents the starting depot and location $n+1$ the ending depot. Let $V=V^{\prime} \cup\{0, n+1\}$ represent the set of all locations. The directed graph $G$ on vertex set $V$ and with arc set $A$ is used to represent our distribution network. Arc set $A$ consists of all arcs leaving the starting depot, $(0, i)$ for $i \in V^{\prime}$, all arcs entering the ending depot, $(i, n+1)$ for $i \in V^{\prime}$, and all arcs between the clients in $V^{\prime}$.

For each directed arc $(i, j) \in A$ a travel $\operatorname{cost} c_{i j}$ and a travel time $t_{i j}$ is given. The travel costs are assumed to be non-negative, $c_{i j} \geq 0$, and to adhere to the triangle inequality, $c_{i j}+c_{j k} \geq c_{i k}$. We assume the same properties for the travel times. Moreover, we require all travel times to be strictly positive, the reason for this is highlighted later.

Let $w_{i}$ be the width of the time window that has to be assigned to client $i \in V^{\prime}$. We refer to this time window as the endogenous time window of client $i$, as opposed to the exogenous time window of client $i$ which defines the interval in which the endogenous time window should be chosen. The exogenous time window of each client $i \in V^{\prime}$ is fixed and given by the interval $\left[s_{i}, e_{i}\right]$, with $e_{i}-s_{i} \geq w_{i}$. Furthermore, the opening hours of the starting depot are given by $\left[s_{0}, e_{0}\right]$ and the opening hours of the ending depot are given by $\left[s_{n+1}, e_{n+1}\right]$.

We assume that we have access to an unlimited number of homogeneous vehicles, each with capacity $Q$. To model demand uncertainty, consider a finite set of possible demand scenarios $\Omega$ and corresponding probabilities $p_{\omega}$ such that $\sum_{\omega \in \Omega} p_{\omega}=1$. The demand of client $i \in V^{\prime}$ in scenario $\omega \in \Omega$ is given by $0<d_{i}^{\omega} \leq Q$.

We now formally state the TWAVRP: find both 1) an assignment of the endogenous 
time windows within the exogenous time windows and 2), for every demand scenario, a routing of the vehicles adhering to the capacity constraints, and consistent with the assigned endogenous time windows, such that the expected routing cost is minimized.

\subsection{Mixed integer linear program}

Next, we present a new mixed integer linear programming formulation for the TWAVRP. First we introduce the decision variables. The time window decisions are given by the continuous variables $y_{i}$ for $i \in V^{\prime}$, which indicate the starting times of the endogenous time windows. That is, the endogenous time window of client $i$ is given by $\left[y_{i}, y_{i}+w_{i}\right]$. As the endogenous time window has to be within the exogenous time window, we require $y_{i} \in\left[s_{i}, e_{i}-w_{i}\right]$.

The vehicle routes are determined by the binary flow variables $x_{i j}^{\omega}$ for $(i, j) \in A$, which are equal to one if a vehicle travels from $i$ to $j$ in scenario $\omega$. The continuous variable $t_{i}^{\omega}$ indicates at what time client $i \in V^{\prime}$ receives delivery in scenario $\omega \in \Omega$.

For notational convenience, we introduce the arc set $\bar{A}$. Let $\bar{A}$ be the set of arcs $A$ to which the $\operatorname{arcs}(i, 0)$ and $(n+1, i)$ have been added for all $i \in V^{\prime}$. To model capacity, similar to Baldacci et al. (2004), we introduce the flow variables $z_{i j}^{\omega}$ for all $(i, j) \in \bar{A}$, $\omega \in \Omega$. Its interpretation depends on the direction the arc is traversed, as given by the $x$-variables. If $z_{i j}^{\omega}$ follows this direction $\left(x_{i j}=1\right)$, it represents the total vehicle load when traveling from client $i$ to client $j$. If it follows the opposite direction $\left(x_{j i}=1\right), z_{i j}^{\omega}$ represents the leftover capacity on the vehicle when traveling from client $j$ to client $i$. If the connection between $i$ and $j$ is not used $\left(x_{i j}=x_{j i}=0\right), z_{i j}^{\omega}$ is zero.

We also introduce the arc sets $A_{i j}$ for all $i, j \in V$. Let $A_{i j}$ be the intersection of $A$ and $\{(i, j),(j, i)\}$, i.e., the arcs between $i$ and $j$ that are in $A$. 
We provide the following mixed integer linear programming formulation:

$$
\begin{aligned}
& \min \sum_{\omega \in \Omega} p_{\omega} \sum_{(i, j) \in A} c_{i j} x_{i j}^{\omega} \\
& \text { s.t. } \sum_{j \in V^{\prime} \cup\{n+1\}} x_{i j}^{\omega}=1 \\
& \sum_{i \in V^{\prime} \cup\{0\}} x_{i j}^{\omega}=1 \quad \forall j \in V^{\prime}, \omega \in \Omega \\
& z_{i j}^{\omega}+z_{j i}^{\omega}=\left(\sum_{(k, l) \in A_{i j}} x_{k l}^{\omega}\right) Q \quad \forall(i, j) \in \bar{A}, i<j, \omega \in \Omega \\
& \sum_{j \in V}\left(z_{j i}^{\omega}-z_{i j}^{\omega}\right)=2 d_{i}^{\omega} \quad \forall i \in V^{\prime}, \omega \in \Omega \\
& \sum_{j \in V^{\prime}} z_{0 j}^{\omega}=\sum_{i \in V^{\prime}} d_{i}^{\omega} \quad \forall \omega \in \Omega \\
& \sum_{j \in V^{\prime}} z_{n+1, j}^{\omega}=\left(\sum_{j \in V^{\prime}} x_{0 j}^{\omega}\right) Q \quad \forall \omega \in \Omega \\
& \sum_{j \in V^{\prime}} z_{j 0}^{\omega}=\left(\sum_{j \in V^{\prime}} x_{0 j}^{\omega}\right) Q-\sum_{i \in V^{\prime}} d_{i}^{\omega} \quad \forall \omega \in \Omega \\
& t_{j}^{\omega}-t_{i}^{\omega} \geq t_{i j} x_{i j}^{\omega}+\left(s_{j}-e_{i}\right)\left(1-x_{i j}^{\omega}\right) \quad \forall i \in V^{\prime}, j \in V^{\prime}, \omega \in \Omega \\
& s_{0}+t_{0 j} \leq t_{j}^{\omega} \quad \forall j \in V^{\prime}, \omega \in \Omega \\
& t_{i}^{\omega}+t_{i, n+1} \leq e_{n+1} \quad \forall i \in V^{\prime}, \omega \in \Omega \\
& t_{i}^{\omega} \geq y_{i} \\
& \forall i \in V^{\prime}, \omega \in \Omega \\
& t_{i}^{\omega} \leq y_{i}+w_{i} \\
& \forall i \in V^{\prime}, \omega \in \Omega \\
& y_{i} \in\left[s_{i}, e_{i}-w_{i}\right] \\
& \forall i \in V^{\prime} \\
& x_{i j}^{\omega} \in \mathbb{B} \\
& \forall(i, j) \in A, \omega \in \Omega \\
& z_{i j}^{\omega} \geq 0
\end{aligned}
$$

The objective (1) is to minimize the expected traveling costs over all scenarios. Constraints (2) and (3) are the flow conservation constraints. Constraints (15) make sure all flows are integral.

Vehicle capacity is modeled by Constraints (4)-(8) and (16), which are based on the 2-commodity flow formulation in Baldacci et al. (2004). Constraints (4) relate opposing arcs: when either $(i, j)$ or $(j, i)$ is used, the corresponding $z$-variables sum to the vehicle capacity.

Constraints (5) can be seen as flow conservation constraints for the $z$-variables: before visiting the client, the load is $d_{i}^{\omega}$ units more than afterwards. After visiting, the empty space is $d_{i}^{\omega}$ units more than before. Hence, the total difference in both flows is equal to $2 d_{i}^{\omega}$. The total vehicle load, capacity and excess capacity in the system are constrained by (6)(8). Constraints (6) set the total vehicle load equal to the total demand of all clients, and Constraints (7) set the total capacity equal to the number of used vehicles multiplied by the vehicle capacity. The total excess capacity of all vehicles leaving the starting depot is set by Constraints (8). Finally, we enforce vehicle capacity to be respected by constraining 
all empty space on the vehicles to be non-negative, as is done in Constraints (16). For more details on these constraints, see Baldacci et al. (2004).

Constraints (9)-(14) deal with the time windows. Constraints (9) are the MTZinequalities that model the time-of-service (Miller et al., 1960). If a vehicle travels from client $i$ to client $j$ in scenario $\omega \in \Omega$, then $x_{i j}^{\omega}=1$ and hence $t_{j}^{\omega}-t_{i}^{\omega} \geq t_{i j}$, i.e., the time-of-service increases with at least the travel time from $i$ to $j$. If no vehicle travels from $i$ to $j$ in scenario $\omega$, then $x_{i j}^{\omega}=0$ and the constraint reads $t_{j}^{\omega}-t_{i}^{\omega} \geq s_{j}-e_{i}$, which always holds as $t_{j}^{\omega} \geq s_{j}$ and $t_{i}^{\omega} \leq e_{i}$. Note that the MTZ-inequalities eliminate cycles, because we have assumed that $t_{i j}>0$ for all $(i, j) \in A$.

Constraints (10) guarantee that vehicles can only leave the starting depot after it opens and Constraints (11) ensure that vehicles arrive at the ending depot before it closes. Constraints (12) and (13) enforce that each client is served within its endogenous time window, while Constraints (14) make sure these endogenous time windows are within the exogenous time windows.

\subsection{Remarks}

In the above formulation, we model capacity using constraints that are based on the 2-commodity flow formulation in Baldacci et al. (2004). The MTZ inequalities are used to model time-of-service. Other formulations for capacity and time-of-service have been considered as well, including several adaptations of models for the CVRP (Baldacci et al., 2004) and the ATSPTW (Ascheuer et al., 2001).

Preliminary testing with all our combinations of capacity constraints and time-ofservice constraints in a branch-and-cut algorithm showed that the best performance is achieved by the combination of the 2-commodity flow formulation to model capacity and the MTZ-inequalities to model time-of-service. This may seem surprising, as in general the MTZ-inequalities typically do not contribute to strong bounds in the LP relaxation. For our instances, however, we have seen that this is compensated for by the relatively strong formulation for capacity.

The MTZ-inequalities require no additional variables and only $n(n+1)|\Omega|$ constraints. This allows for a branch-and-cut strategy in which we process the nodes of the search tree faster than in all other alternatives we considered. Using the 2-commodity flow formulation to model capacity yields a good trade-off between the number of variables and constraints, and the strength of the formulation.

\section{Branch-and-cut algorithm}

In this section, we present our branch-and-cut algorithm. First, we present valid inequalities from the literature, which we use to strengthen the LP-bound of (1)-(16). Next, we introduce our branching strategy. In Section 4 we separately introduce the novel precedence inequalities.

\section{$3.1 \quad 2$-cycle elimination}

The current mixed integer linear program ensures that no integer feasible solution contains cycles. Explicit cycle elimination, however, may still strengthen the LP-bound.

As there are only a quadratic number of 2-cycles in a given graph, we can eliminate all 2-cycles with a relatively small number of constraints. We do so by adding all of the 
following inequalities to the formulation:

$$
x_{i j}^{\omega}+x_{j i}^{\omega} \leq 1 \forall i \in V^{\prime}, j \in V^{\prime}, \omega \in \Omega .
$$

\subsection{Rounded capacity inequalities}

The capacity inequalities are well-known valid inequalities for the VRPTW, and thus may be applied directly to the TWAVRP per scenario. Let $\lambda_{S}^{\omega}$ be the minimum number of vehicles required to satisfy the demand of all clients in $S \subseteq V^{\prime}$ in scenario $\omega$. The capacity inequalities are given by

$$
\sum_{(i, j) \in A \mid i \in S, j \in V \backslash S} x_{i j}^{\omega} \geq \lambda_{S}^{\omega} \forall S \subseteq V^{\prime},|S| \geq 2, \omega \in \Omega .
$$

That is, we require for each subset $S \subseteq V^{\prime}$ that the total number of vehicles leaving $S$ is sufficient to satisfy all demand in $S$.

Calculating $\lambda_{S}^{\omega}$ requires solving a bin-packing problem, which is NP-hard in general. Hence, as is commonly done, we only consider the weakened inequalities in which $\lambda_{S}^{\omega}$ is replaced by the bin-packing lower bound $\left\lceil\left(\sum_{i \in S} d_{i}^{\omega}\right) / Q\right\rceil$. These valid inequalities are known as the rounded capacity inequalities. Simply adding all rounded capacity inequalities is not efficient, but when used in a branch-and-cut algorithm, they can be very effective (Baldacci et al., 2012).

We add the following $|\Omega|$ inequalities to the formulation:

$$
\sum_{i \in V^{\prime}} x_{i, n+1}^{\omega} \geq\left\lceil\frac{\sum_{i \in V^{\prime}} d_{i}^{\omega}}{Q}\right\rceil \forall \omega \in \Omega
$$

The other rounded capacity inequalities are separated and added in a cutting plane fashion. We use the separation algorithm from Lysgaard (2003). Details on this algorithm can be found in Lysgaard et al. (2004).

\subsection{Branching strategy}

In the formulation in Section 2.1, we require each $x_{i j}^{\omega}$ to be binary. However, we show that it is sufficient to require that all $x_{i j}^{\omega} \in[0,1]$, that $x_{i j}^{\omega}$ is binary for all arcs connected to one of the depots, and furthermore that $x_{i j}^{\omega}+x_{j i}^{\omega}$ is binary for all $i, j \in V^{\prime}(i \neq j)$. We define the following integrality conditions:

$$
\begin{gathered}
x_{0 j}^{\omega} \in \mathbb{B} \forall j \in V^{\prime}, \omega \in \Omega, \\
x_{i, n+1}^{\omega} \in \mathbb{B} \forall i \in V^{\prime}, \omega \in \Omega, \\
x_{i j}^{\omega}+x_{j i}^{\omega} \in \mathbb{B} \forall i<j, i \in V^{\prime}, j \in V^{\prime}, \omega \in \Omega, \\
x_{i j}^{\omega} \in[0,1] \forall(i, j) \in A, \omega \in \Omega .
\end{gathered}
$$

Proposition 1. All integer feasible solutions to (1)-(14), (16), (17), (19), and (20)-(23) satisfy

$$
x_{i j}^{\omega} \in \mathbb{B} \forall(i, j) \in A, \omega \in \Omega .
$$

Proof. See Appendix A. 
Proposition 1 suggests that for any $i, j \in V^{\prime}$ instead of branching on whether a single directed arc is used, we may also branch on whether a connection between $i$ and $j$ (regardless of direction) is used. This decision can be made per connection, as $x_{i j}^{\omega} \in \mathbb{B}$ and $x_{j i}^{\omega} \in \mathbb{B}$ together imply $x_{i j}^{\omega}+x_{j i}^{\omega} \in \mathbb{B}, x_{i j}^{\omega} \in[0,1]$ and $x_{j i}^{\omega} \in[0,1]$. That is, Proposition 1 is still applicable if for some connections we branch on both $x_{i j}^{\omega}$ and $x_{j i}^{\omega}$ and for the other connections we branch on $x_{i j}^{\omega}+x_{j i}^{\omega}$.

Branching on $x_{i j}$ means that we partition the feasible region in two parts. In the first part it is assumed that $j$ is visited directly after $i$ by the same vehicle. In the other part we assume $j$ is not visited directly after $i$. Knowing whether $j$ is visited directly after $i$ has important implications for the time-of-service variables $t_{i}^{\omega}$ and $t_{j}^{\omega}$. The value $\left(s_{j}-e_{i}\right)$ in the MTZ-inequalities is essentially a big-M, hence fractional values of the flow variables cause the time constraints to be very weak or inactive. When $x_{i j}^{\omega}=1$, however, we have $t_{j}^{\omega} \geq t_{i}^{\omega}+t_{i j}$. Especially when $t_{i j}$ is big, this inequality has a big effect on the time-of-service variables.

If the value of $t_{i j}$ is close to zero, this argument no longer holds, whether $x_{i j}=1$ or $x_{j i}=1$ is not that important for the time-of-service variables. For the capacity constraints it is important to know whether $i$ and $j$ are visited by the same vehicle, but it is less important to know in which order this happens. Hence, it makes sense to branch on $x_{i j}+x_{j i}$ to split the feasible region in two parts: one part in which $j$ is visited directly after $i$, or the other way around, and one part in which there is no direct connection between $i$ and $j$.

To take these effects into account, we introduce the parameter $\rho \in[0,1]$. Then, for the fraction $\rho$ of all arcs with the shortest travel time, we branch on the connections. For the other arcs, we branch on $x_{i j}^{\omega}$ and $x_{j i}^{\omega}$ separately. Note that $\rho=0$ corresponds to always branching on individual arcs. If $\rho=1$, we always branch on connections. By varying $\rho$, a good compromise can be found between the number of variables and the strength of the LP relaxation.

\section{Precedence inequalities}

In this section, we present a novel set of valid inequalities, the precedence inequalities, in which each inequality involves multiple scenarios. First, we make some important observations.

If we want to visit first $i$ and later $j$, both within their respective time windows, the time between the two visits is bounded. Let $\bar{t}_{i j}$ be the maximum time between these visits, for all $i, j \in V^{\prime}$. That is, we define $\bar{t}_{i j}=\left(y_{j}+w_{j}\right)-y_{i}$ and similarly $\bar{t}_{j i}=\left(y_{i}+w_{i}\right)-y_{j}$, as illustrated in Figure 1. It follows that $\bar{t}_{i j}+\bar{t}_{j i}=w_{i}+w_{j}$.

Now consider a solution to the TWAVRP for which in scenario $\omega$ there is a route visiting first $i$, and later $j$. Furthermore, assume there is a different scenario $\omega^{\prime}$ with a route visiting first $j$, and later $i$. It follows that the time taken to get from $i$ to $j$ in one scenario, plus the time taken to get from $j$ to $i$ in another scenario, can be at most the sum of the widths of the time windows, $w_{i}+w_{j}$.

We formalize this in Observation 2. Let $A_{p}$ be the set of arcs used by path $p$ in $G$ and let $\mathscr{P}_{i j}$ be the set of all elementary paths in $G$ starting at $i \in V$ and ending at $j \in V$.

Observation 2. For given vertices $i, j \in V^{\prime}(i \neq j)$, for any integer feasible solution to the TWAVRP in which both path $p \in \mathscr{P}_{i j}$ is used in scenario $\omega \in \Omega$ and path $q \in \mathscr{P}_{j i}$ is 


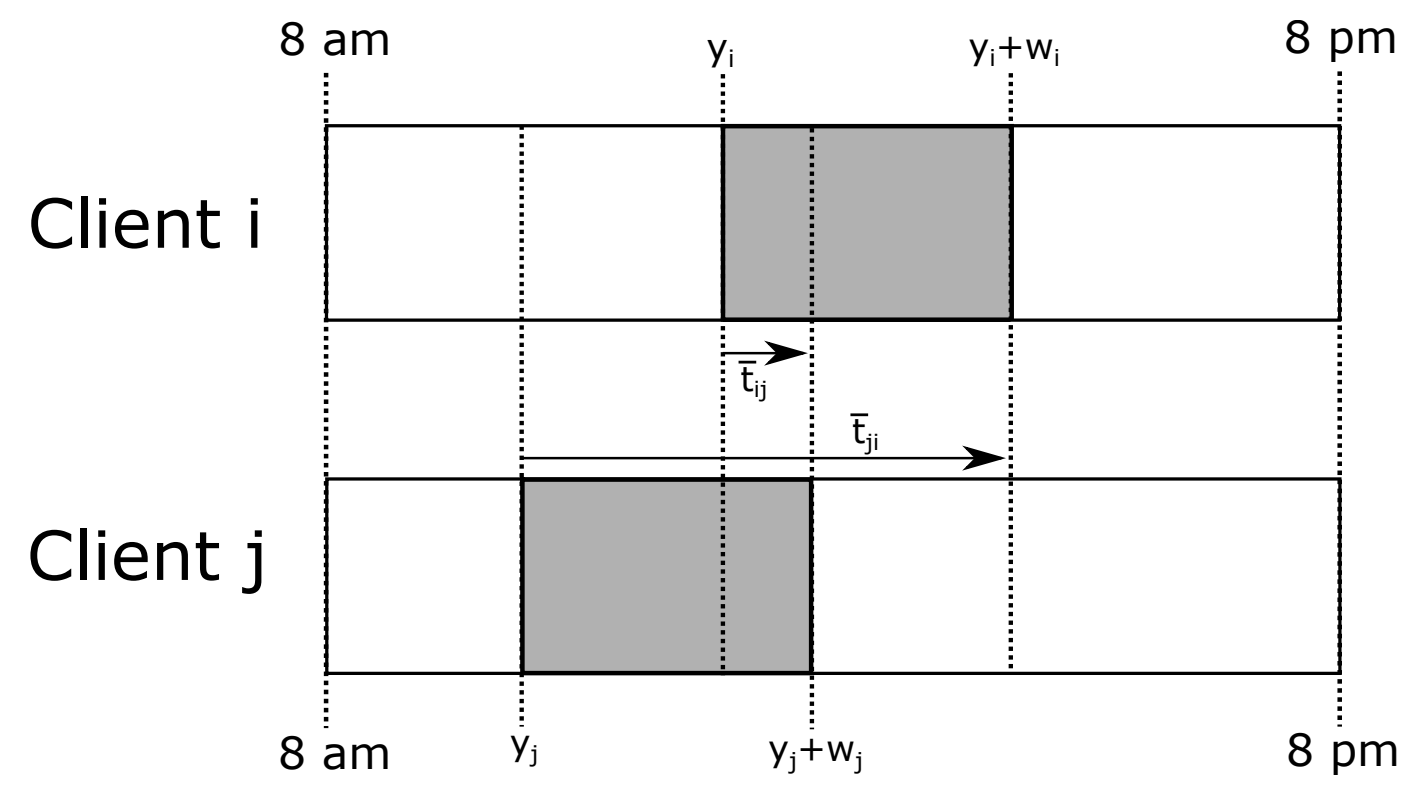

Figure 1: Example time window assignment and maximum allowed driving times.

used in scenario $\omega^{\prime} \in \Omega$ the following holds:

$$
\sum_{(k, l) \in A_{p}} t_{k l}+\sum_{(k, l) \in A_{q}} t_{k l} \leq w_{i}+w_{j}
$$

To construct valid inequalities based on Observation 2, we make use of Theorem (4.5) in Ascheuer et al. (2000), which we restate for the TWAVRP as the following lemma.

Lemma 3. For any integer feasible TWAVRP solution, a set of clients $S \subseteq V^{\prime}$ and two vertices $i, j \in V^{\prime} \backslash S(i \neq j)$, a single vehicle visits $i$ first, then all clients in $S$ and then $j$ consecutively in scenario $\omega \in \Omega$ if and only if:

$$
\sum_{l \in S} x_{i l}^{\omega}+\sum_{k \in S} \sum_{l \in S} x_{k l}^{\omega}+\sum_{k \in S} x_{k j}^{\omega}+x_{i j}^{\omega}=|S|+1
$$

Lemma 3 gives us a criterion for testing whether there is a path visiting client $i$, then visiting a subset of other clients, and then visiting client $j$. Combining this lemma with Observation 2 allows us to formulate the precedence inequalities.

Let us denote by $(S: T)$ the set of arcs in $A$ which start in $S$ and end in $T$, for $S$ and $T$ vertices or sets of vertices. For notational convenience we introduce the sets $\mathcal{S}(i, j)=\left\{S \mid S \subseteq V^{\prime} \backslash\{i, j\}\right\}$ for all $i, j \in V^{\prime}$. That is, $\mathcal{S}(i, j)$ is the set of all possible subsets of clients not containing clients $i$ and $j$. When traveling from $i$ to $j$, visiting exclusively clients from $S$, only the arcs in $(i: S) \cup(S: S) \cup(S: j) \cup(i: j)$ are relevant. Therefore, we introduce $\mathcal{F}(i, S, j)=\{F \mid F \subseteq(i: S) \cup(S: S) \cup(S: j) \cup(i: j)\}$, which for given $i, j \in V^{\prime}$ and $S \in \mathcal{S}(i, j)$ is the set of all possible subsets of these arcs.

Furthermore, let $\delta_{i j}(S, F)$ be the shortest possible travel time from client $i \in V^{\prime}$ to client $j \in V^{\prime}$, visiting all clients in $S \in \mathcal{S}(i, j)$ in between, using only arcs from set $F \in \mathcal{F}(i, S, j)$. If no such path exists $\delta_{i j}(S, F)=\infty$. We then arrive at the main theorem for the precedence inequalities:

Theorem 4. Precedence inequalities: For given scenarios $\omega, \omega^{\prime} \in \Omega\left(\omega \neq \omega^{\prime}\right)$, given clients $i, j \in V^{\prime}(i \neq j)$, given vertex set $S \in \mathcal{S}(i, j)$, corresponding to clients visited in 
scenario $\omega$, and vertex set $S^{\prime} \in \mathcal{S}(j, i)$ corresponding to clients visited in scenario $\omega^{\prime}$, and given arc sets $F \in \mathcal{F}(i, S, j)$ and $F^{\prime} \in \mathcal{F}\left(j, S^{\prime}, i\right)$ such that $\delta_{i j}(S, F)+\delta_{j i}\left(S^{\prime}, F^{\prime}\right)>w_{i}+w_{j}$, the following are valid inequalities:

$$
\sum_{(k, l) \in F} x_{k l}^{\omega}+\sum_{(k, l) \in F^{\prime}} x_{k l}^{\omega^{\prime}} \leq|S|+\left|S^{\prime}\right|+1 .
$$

Proof. This is a direct application of Observation 2. Lemma 3 shows that Observation 2 is contradicted if and only if $\sum_{(k, l) \in F} x_{k l}^{\omega}+\sum_{(k, l) \in F^{\prime}} x_{k l}^{\omega^{\prime}}=|S|+\left|S^{\prime}\right|+2$. By integrality of the $x$-variables, the theorem follows.

It is possible to generalize this result by redefining $\delta_{i j}(S, F)$ to be the minimum travel time to visit client $i$, all clients in $S$ and then client $j$ using only arcs of $F$, but only using paths that can be feasible when considering the exogenous time windows. We choose not to present this generalization, as we consider an application in which the exogenous time windows are in general very wide. The proposed generalization is then unlikely to add much value, while making it more complex to identify violated inequalities.

Clearly, the number of possible precedence inequalities is exponential in the number of clients. Hence, it is not efficient to add all precedence inequalities to the formulation directly. Instead, we separate the precedence inequalities in a cutting plane fashion. Note that exact separation of the precedence inequalities is difficult, as finding violated precedence inequalities is co-NP-hard, which is proven in Appendix B. For this reason, we separate subsets of the precedence inequalities exactly, and we present heuristics for more general precedence inequalities.

Before we consider these subsets, we state the following two lemmas, which will be useful when deriving our separation algorithms. We provide a lower bound on the flow in $F$ for violated inequalities and we show that for every violated inequality $F$ is cyclic or $F$ contains an elementary $(i, j)$-path visiting all vertices in $S$.

Lemma 5. Let $i, j \in V^{\prime}, S \in \mathcal{S}(i, j), S^{\prime} \in \mathcal{S}(j, i), F \in \mathcal{F}(i, S, j)$ and $F^{\prime} \in \mathcal{F}\left(j, S^{\prime}, i\right)$ correspond to a violated precedence inequality, for a feasible solution to the LP relaxation of the formulation (1)-(16), (17) and (19). Then both

$$
\sum_{(k, l) \in F} x_{k l}^{\omega}>|S|
$$

and

$$
\sum_{(k, l) \in F^{\prime}} x_{k l}^{\omega^{\prime}}>\left|S^{\prime}\right|
$$

Proof. By definition of $\mathcal{F}(i, S, j)$ all directed arcs in $F$ point to vertex $j$, or to a vertex in $S$. Thus, by the flow conservation constraints it follows that the total flow in $F$ is bounded by $|S|+1$. Hence, we have $\sum_{(k, l) \in F} x_{k l}^{\omega} \leq|S|+1$ and similarly $\sum_{(k, l) \in F^{\prime}} x_{k l}^{\omega^{\prime}} \leq\left|S^{\prime}\right|+1$. From Theorem 4 it follows that for a violated precedence inequality $\sum_{(k, l) \in F} x_{k l}^{\omega}+\sum_{(k, l) \in F^{\prime}} x_{k l}^{\omega^{\prime}}>$ $|S|+\left|S^{\prime}\right|+1$. Combining these facts proves the lemma.

Lemma 6. Let $i, j \in V^{\prime}, S \in \mathcal{S}(i, j), S^{\prime} \in \mathcal{S}(j, i), F \in \mathcal{F}(i, S, j)$ and $F^{\prime} \in \mathcal{F}\left(j, S^{\prime}, i\right)$ correspond to a violated precedence inequality, for a feasible solution to the $L P$ relaxation of the formulation (1)-(16), (17) and (19). Then $F$ contains a cycle or $F$ contains an elementary $(i, j)$-path through all vertices of $S$. Also $F^{\prime}$ contains a cycle or $F^{\prime}$ contains an elementary $(j, i)$-path through all vertices of $S^{\prime}$. 
Proof. We prove this statement for $F$, as for $F^{\prime}$ the proof is analogous. If $F$ contains a cycle, the lemma holds. Next, we assume $F$ is acyclic. Moreover, we assume that $F$ does not contain an elementary path from $i$ to $j$ through all vertices of $S$, and we show that this leads to a contradiction.

Because $F$ is acyclic, the vertices of $S$ can be relabeled $v_{1}, v_{2}, \ldots, v_{|S|}$ such that if $l<k$ then $\left(v_{k}, v_{l}\right) \notin F$ (see Kahn (1962)). By assumption, there is no elementary path from $i$ through all $v_{1}, v_{2}, \ldots, v_{|S|}$ to $j$. Hence, there exists an integer $g \in\{1,2, \ldots,|S|-1\}$ such that there is no arc from $v_{g}$ to $v_{g+1}$.

Let $U_{1}=\left\{i, v_{1}, v_{2}, \ldots, v_{g-1}\right\}$ and let $U_{2}=\left\{v_{g+2}, v_{g+3}, \ldots, v_{|S|}, j\right\}$. By construction, we have that

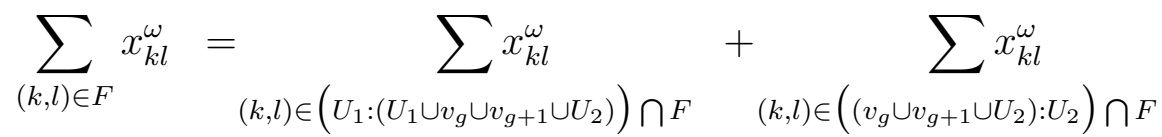

$$
\begin{aligned}
& \leq\left|U_{1}\right|+\left|U_{2}\right|=|S| \text {. }
\end{aligned}
$$

This follows because the total outflow of the vertices in $U_{1}$ is bounded by $\left|U_{1}\right|$ due to the flow conservation constraints. Similarly, the total inflow of the vertices in $U_{2}$ is bounded by $\left|U_{2}\right|$. Hence, it follows that the total flow captured by $F$ is bounded by $\left|U_{1}\right|+\left|U_{2}\right|=|S|$.

This contradicts Lemma 6 , which states that $\sum_{(k, l) \in F} x_{k l}^{\omega}>|S|$. Thus, our assumption that $F$ does not contain an elementary path from $i$ through $S$ to $j$ is false. Hence, we have proven that $F$ contains a cycle, or contains an elementary $(i, j)$-path visiting all clients in $S$.

\subsection{Path precedence inequalities}

The first subset of precedence inequalities we consider, is the subset for which $F$ and $F^{\prime}$ both form a single elementary path, which we refer to as the path precedence inequalities. We make use of the following proposition:

Proposition 7. For any integer feasible solution to the TWAVRP:

$$
\begin{gathered}
\sum_{(k, l) \in A_{p}} t_{k l}+\sum_{(k, l) \in A_{q}} t_{k l}>w_{i}+w_{j} \Longrightarrow \sum_{(k, l) \in A_{p}} x_{k l}^{\omega}+\sum_{(k, l) \in A_{q}} x_{k l}^{\omega^{\prime}} \leq\left|A_{p}\right|+\left|A_{q}\right|-1 \\
\forall(i, j) \in A, p \in \mathscr{P}_{i j}, q \in \mathscr{P}_{j i}, \omega \in \Omega, \omega^{\prime} \in \Omega .
\end{gathered}
$$

Proof. This is a direct application of Theorem 4 with $F=A_{p}$ and $F^{\prime}=A_{q}$. Note that $\delta_{i j}(S, F)=\sum_{(k, l) \in A_{p}} t_{k l}$, as following the path $p$ is the only way to visit all vertices in $S$ using only vertices in $F$. Analogously, $\delta_{j i}\left(S^{\prime}, F^{\prime}\right)=\sum_{(k, l) \in A_{q}} t_{k l}$. Finally, note that $|S|=\left|A_{p}\right|-1$ and $\left|S^{\prime}\right|=\left|A_{q}\right|-1$, and hence $|S|+\left|S^{\prime}\right|+1=\left|A_{p}\right|+\left|A_{q}\right|-1$.

Proposition 7 defines valid inequalities for paths only, instead of for arbitrary sets of vertices and arcs. Next, we show some properties of the path precedence inequalities. These properties are then used to prove that for a given solution to the LP relaxation, all violated path precedence inequalities can be found in polynomial time.

Lemma 8. All violated path precedence inequalities adhere to the following two inequalities:

$$
\sum_{(k, l) \in A_{p}} x_{k l}^{\omega}>\left|A_{p}\right|-1
$$




$$
\sum_{(k, l) \in A_{q}} x_{k l}^{\omega^{\prime}}>\left|A_{q}\right|-1 .
$$

Proof. This is a direct application of Lemma 5 with $F=A_{p}$ and $F^{\prime}=A_{q}$.

Lemma 9. Let $p$ and $q$ correspond to a violated path precedence inequality. Path $p$ in graph $G$ contains at most one arc $(k, l)$ for which $x_{k l}^{w} \leq \frac{1}{2}$. Path $q$ contains at most one $\operatorname{arc}\left(k^{\prime}, l^{\prime}\right)$ for which $x_{k^{\prime} l^{\prime}}^{\omega^{\prime}} \leq \frac{1}{2}$.

Proof. Suppose $p$ has $m \geq 2$ arcs for which $x_{k l}^{\omega} \leq \frac{1}{2}$. This implies $\sum_{(k, l) \in A_{p}} x_{k l}^{\omega} \leq\left|A_{p}\right|-$ $\frac{1}{2} m \leq\left|A_{p}\right|-1$. Hence (31) is not satisfied. It follows that $p$ has at most one arc for which $x_{k l}^{\omega} \leq \frac{1}{2}$. The proof for path $q$ is analogous.

Proposition 10. All violated path precedence inequalities can be found in polynomial time.

Proof. To find all violated path precedence inequalities, we generate an exhaustive list of candidate paths from $i$ to $j$ which meet the necessary condition given by Lemma 9 . If we do the same for all candidate paths from $j$ to $i$, we can check for all combinations of the candidates whether (30) is violated.

To generate a list of candidates, we first use Lemma 9, which states that for scenario $\omega \in \Omega$ a candidate uses at most one arc for which $x_{k l}^{\omega} \leq \frac{1}{2}$. Starting at $i$, the path thus first uses a (possibly zero) number of arcs for which $x_{k l}^{\omega}>\frac{1}{2}$, followed by zero or one arcs for which $x_{k l}^{\omega} \leq \frac{1}{2}$. After that, we visit another (possibly zero) number of arcs for which $x_{k l}^{\omega}>\frac{1}{2}$ before we reach $j$.

By the flow conservation constraints, the total outflow and the total inflow of a vertex are both equal to one. Hence, at each vertex there can be at most one incoming arc and one outgoing arc for which $x_{k l}^{\omega}>\frac{1}{2}$. This implies there is at most one elementary path leaving $i$ for which all arcs have an $x$ value larger than $\frac{1}{2}$. Analogously there is at most one elementary path entering $j$ for which all $x$ values are larger than $\frac{1}{2}$. Finding these two elementary paths takes $O\left(n^{2}\right)$ time, as the paths contain $O(n)$ vertices, and, for a single vertex, determining which arc has value larger than $\frac{1}{2}$ takes $O(n)$ time.

All candidate paths from $i$ to $j$ can thus be constructed by starting in $i$, following the arcs with $x$ values larger than $\frac{1}{2}$ up to a certain point after which an arc with $x$ value less or equal to $\frac{1}{2}$ is taken to arrive at the path of arcs with $x$ values larger than $\frac{1}{2}$ that arrives at $j$, which is followed until we reach $j$. That is, without loss of generality we sequentially visit the vertices $i=v_{1}, v_{2}, \ldots, v_{f}, w_{g}, w_{g-1}, \ldots, w_{1}=j$ for integers $f$ and $g$ between 1 and $n$.

For given $f$ and $g$, the total flow of the candidate path is given by $\sum_{i=1}^{f-1} x_{v_{i} v_{i+1}}+x_{v_{f} w_{g}}+$ $\sum_{i=1}^{g-1} x_{w_{i+1} w_{i}}$, and the total travel time is given by $\sum_{i=1}^{f-1} t_{v_{i} v_{i+1}}+t_{v_{f} w_{g}}+\sum_{i=1}^{g-1} t_{w_{i+1} w_{i}}$. By precalculating the summations for all $f$ and $g$ in $O\left(n^{2}\right)$ time, this part only takes constant time.

For each scenario, there are $O\left(n^{2}\right)$ combinations of $f$ and $g$. We thus find $O\left(|\Omega| n^{2}\right)$ candidates from $i$ to $j$. Then, we check for all combinations of candidates from $i$ to $j$ and candidates from $j$ to $i$ if the condition in Proposition 7 is satisfied. Per combination, this takes constant time, as we only sum the predetermined values of total flow and total travel time. There are $O\left(\left(|\Omega| n^{2}\right)^{2}\right)$ such combinations. As we repeat the procedure for all combinations of two vertices $i$ and $j$, the total time complexity is $O\left(|\Omega|^{2} n^{6}\right)$. 


\subsection{Tournament precedence inequalities}

In the previous section, we have introduced the path precedence inequalities, which are precedence inequalities based on elementary paths. In this section we present a broader subset of the precedence inequalities, in which both $(S, F)$ and $\left(S^{\prime}, F^{\prime}\right)$ represent a directed acyclic graph. Furthermore, we show that to satisfy all these valid inequalities it is sufficient to restrict ourselves to those directed acyclic graphs $F$ and $F^{\prime}$ obtained by taking the transitive closures of an elementary path. The transitive closure of a set of $\operatorname{arcs} F \subseteq A$ in graph $G$, is defined as follows:

$$
\operatorname{trcl}(F):=\{(k, l) \in A: l \text { can be reached from } k \text { using only arcs in } F\} .
$$

These inequalities are similar to the tournament constraints of Ascheuer et al. (2000), hence we call this class the tournament precedence inequalities. In Ascheuer et al. (2000), the tournament inequalities are introduced for the ATSPTW, which are obtained by bounding the total flow on the transitive closure of a simple path which violates (exogenous) time window constraints or cannot be extended without violating time window constraints. Next we present the tournament precedence inequalities, discuss how to separate them, and show that if all tournament precedence inequalities are satisfied then so are all precedence inequalities based on directed acyclic graphs.

Proposition 11. For any integer feasible solution to the TWAVRP:

$$
\begin{gathered}
\sum_{(k, l) \in A_{p}} t_{k l}+\sum_{(k, l) \in A_{q}} t_{k l}>w_{i}+w_{j} \Longrightarrow \sum_{(k, l) \in \operatorname{trcl}\left(A_{p}\right)} x_{k l}^{\omega}+\sum_{(k, l) \in \operatorname{trcl}\left(A_{q}\right)} x_{k l}^{\omega^{\prime}} \leq\left|A_{p}\right|+\left|A_{q}\right|-1 \\
\forall(i, j) \in A, p \in \mathscr{P}_{i j}, q \in \mathscr{P}_{j i}, \omega \in \Omega, \omega^{\prime} \in \Omega .
\end{gathered}
$$

Proof. This is a direct application of Theorem 4 with $F=\operatorname{trcl}\left(A_{p}\right)$ and $F^{\prime}=\operatorname{trcl}\left(A_{q}\right)$. Observe that taking the transitive closure of an elementary path yields a directed acyclic graph, which contains only a single path visiting all vertices. In particular, $\delta_{i j}(S, F)=$ $\delta_{i j}\left(S, \operatorname{trcl}\left(A_{p}\right)\right)=\delta_{i j}\left(S, A_{p}\right)=\sum_{(k, l) \in A_{p}} t_{k l}$. Similarly, $\delta_{j i}(S, F)=\sum_{(k, l) \in A_{q}} t_{k l}$. Note that $|S|=\left|A_{p}\right|-1$ and $\left|S^{\prime}\right|=\left|A_{q}\right|-1$, and hence $|S|+\left|S^{\prime}\right|+1=\left|A_{p}\right|+\left|A_{q}\right|-1$.

Corollary 12. If a path precedence inequality is violated, then its corresponding tournament precedence inequality (by taking transitive closures) is violated as well.

Proof. Follows directly from Proposition 11, the non-negativity of the $x$ variables and $F \subseteq \operatorname{trcl}(F)$ for all $F \subseteq A$.

As a result, we can find violated tournament precedence inequalities by separating path precedence inequalities. However, not all violated tournament precedence inequalities can be found in this way. Hence, to separate all tournament precedence inequalities, we next present another algorithm.

First, per scenario, we make a list of all elementary paths in $G$, not involving the depot vertices. By definition, each tournament precedence inequality is characterized by two elementary paths and two scenarios. Hence, after we generate the lists, we can separate the tournament precedence inequalities by combining elementary paths from the lists, and checking the condition given in Proposition 11 for each pair.

To construct the list per scenario we use a procedure similar to that described in Ascheuer et al. (2001) to detect violated tournament constraints for the ATSPTW. We 
enumerate all paths but backtrack as soon as $\sum_{(k, l) \in \operatorname{trcl}\left(A_{p}\right)} x_{k l}^{\omega} \leq\left|A_{p}\right|-1$. It is suggested in Ascheuer et al. (2001) that only a polynomial number of paths is generated this way, which would imply that our separation routine, involving multiple scenarios, also requires only a polynomial number of iterations.

We have mentioned that, for separating tournament precedence inequalities, restricting to transitive closures of elementary paths still allows us to capture all precedence inequalities based on directed acyclic graphs. We state this formally in the following lemma.

Lemma 13. Let $i, j \in V^{\prime}, S \in \mathcal{S}(i, j), S^{\prime} \in \mathcal{S}(j, i), F \in \mathcal{F}(i, S, j)$ and $F^{\prime} \in \mathcal{F}\left(j, S^{\prime}, i\right)$ correspond to a precedence inequality, for a feasible solution to the LP relaxation of the formulation (1)-(16), (17) and (19). Furthermore, assume $F$ and $F^{\prime}$ are acyclic. If all tournament precedence inequalities are satisfied, then this precedence inequality is also satisfied.

Proof. As $F$ is assumed to be acyclic, by Lemma 6 it contains an elementary path $p \in \mathcal{P}_{i j}$ through all vertices of $S$. By definition of the transitive closure, it follows that $F \subseteq$ $\operatorname{trcl}\left(A_{p}\right)$. Analogously we have $F^{\prime} \subseteq \operatorname{trcl}\left(A_{q}\right)$ for some $q \in \mathcal{P}_{j i}$ visiting all vertices of $S^{\prime}$.

We have $\sum_{(k, l) \in F} x_{k l}^{\omega}+\sum_{(k, l) \in F^{\prime}} x_{k l}^{\omega^{\prime}} \leq \sum_{(k, l) \in \operatorname{trcl}\left(A_{p}\right)} x_{k l}^{\omega}+\sum_{(k, l) \in \operatorname{trcl}\left(A_{q}\right)} x_{k l}^{\omega^{\prime}} \leq|S|+\left|S^{\prime}\right|+$ 1 , as all tournament precedence inequalities are assumed to be satisfied. It follows that if all tournament precedence inequalities are satisfied, each precedence inequality based on directed acyclic graphs is satisfied as well.

\subsection{Additional strategies}

We have discussed two subsets of the precedence inequalities with corresponding separation strategies. There are, however, some additional strategies that can be utilized.

Note that both the separation algorithm for the path precedence inequalities and the separation algorithm for the tournament precedence inequalities first generate a list of viable candidates $i, j \in V, S \in \mathcal{S}(i, j)$ and $F \in \mathcal{F}(i, S, j)$ per scenario, after which all combinations of candidates are checked to find violated inequalities. An additional strategy is to opportunistically alter these candidates when combining them to create stronger inequalities.

Recall that one way to do this, is by separating path precedence inequalities and taking transitive closures, resulting in tournament precedence inequalities (Corollary 12). Another strategy is to complete $F$ by adding arcs. That is, let $F^{*}$ be the maximum cardinality element of $\mathcal{F}(i, S, j)$. By definition, $F^{*}=(i: S) \cup(S: S) \cup(S: j)$. Note that $F^{*}$ is not acyclic, and hence in general $\delta_{i j}\left(S, F^{*}\right) \neq \delta_{i j}(S, F)$. Furthermore, $\delta_{i j}\left(S, F^{*}\right)$ is hard to calculate.

Therefore, we introduce an easy to calculate lower bound on $\delta_{i j}\left(S, F^{*}\right)$. Note that any violated precedence inequality found while using this lower bound is valid for the actual value of $\delta_{i j}\left(S, F^{*}\right)$ as well. It is well known that the weights of a minimum spanning tree can be used as a lower bound on the length of the shortest elementary path visiting all vertices. It thus follows that:

$$
\delta_{i j}\left(S, F^{*}\right) \geq \min _{k \in S}\left\{t_{i k}\right\}+\operatorname{MST}(S)+\min _{k \in S}\left\{t_{k j}\right\},
$$

in which $\operatorname{MST}(S)$ represents the weight of the minimum weight spanning tree of an undirected complete graph with vertex set $S$ and edge weight $\min \left\{t_{k l}, t_{l k}\right\}$ for each edge $(k, l)$. 
We consider the following strategies. First, separate either path precedence inequalities or tournament precedence inequalities. The path precedence inequalities may be converted to tournament precedence inequalities. Each resulting tournament precedence inequality corresponds to sets $i, j \in V^{\prime}, S \in \mathcal{S}(i, j), F \in \mathcal{F}(i, S, j), S^{\prime} \in \mathcal{S}(j, i)$ and $F \in \mathcal{F}(j, S, i)$. Now try whether a violated precedence inequality can be obtained by replacing $F$ by $F^{*}$ and/or $F^{\prime}$ by $F^{\prime *}$, using the lower bounds on travel time given by (35). If so, use these stronger valid inequalities.

\section{$5 \quad$ Numerical experiments}

Next, we present the results of our numerical experiments to test the effectiveness of our new formulation, the precedence inequalities and the branching strategy. Furthermore, we present experiments in which our algorithm is compared to the branch-price-and-cut algorithm of Spliet and Gabor (2014).

All experiments are run on an Intel i7 $3.5 \mathrm{GHz}$ computer with 16GB of RAM. To allow for a fair comparison between algorithms, we restrict all experiments to a single thread on a single core. As a basis for our implementation, we use the commercial solver CPLEX version 12.5, with default settings. We disable all CPLEX's built in valid inequalities, so we can more accurately test the effect of the valid inequalities discussed in this paper.

Our own valid inequalities will be generated in a callback, which is called each time the LP relaxation has been solved, or re-solved after adding valid inequalities. In this callback we separate rounded capacity inequalities and precedence inequalities, and only afterwards the LP is resolved. We use the built-in 'traditional branch-and-cut' in combination with our own branching strategy as discussed in Section 3.

For our branch-and-cut algorithm we use the 64 bit version of CPLEX, which allows for the full 16GB of memory to be used. The algorithm of Spliet and Gabor (2014) requires less memory, and hence we use the 32 bit version of CPLEX, which gives a slightly better performance.

We use a one hour time limit per instance in all experiments. From preliminary tests we have found that almost every instance is unsolvable within the time limit without separating rounded capacity inequalities, so we separate those in all experiments.

\subsection{Test-instances}

First, we introduce the different sets of test-instances which we use for our numerical experiments.

\subsubsection{Small instances}

We use forty instances introduced by Spliet and Gabor (2014). These instances are randomly generated instances, inspired by a Dutch retail chain. The set contains ten instances of 10, 15, 20 and 25 clients respectively. The clients are uniformly distributed over a square with sides of length five. Both the starting depot and the ending depot are located in the center of the square. The travel cost and the travel time in hours between two points in the square is equal to the Euclidean distance.

Each instance includes three demand scenarios, each with equal probability of occurrence. The average demand is about $1 / 6$ vehicle load. The exogenous time windows 
are rather wide: on average the exogenous time window of the client has width 10.8 , compared to an endogenous time window width of 2 .

\subsubsection{Large instances}

To be able to test our branch-and-cut algorithm on larger instances as well, we have generated fifty additional instances in the same way that the small instances have been generated. That is, we created ten instances of 30, 35, 40, 45 and 50 clients respectively. All instances are available online.

\subsection{Branch-and-cut experiments}

Next, we compare the branching strategies and the separation algorithms for the precedence inequalities, using the forty small instances. We consider six different strategies to separate precedence inequalities, which have been detailed in Section 4.3:

$\mathbf{N} \quad$ Do not separate precedence inequalities.

$\mathbf{P} \quad$ Separate path precedence inequalities.

P2T 1) Separate path precedence inequalities.

2) Turn them into tournament precedence inequalities.

$\mathbf{P 2 C}$ 1) Separate path precedence inequalities.

2) Turn them into tournament precedence inequalities.

3) Complete $F$ and/or $F^{\prime}$ by adding additional arcs and add corresponding violated precedence inequalities if they are found.

T Separate tournament precedence inequalities.

T2C 1) Separate tournament precedence inequalities.

2) Complete $F$ and/or $F^{\prime}$ by adding additional arcs and add corresponding violated precedence inequalities if they are found.

For the branching strategy, we conduct experiments for $\rho \in\{0,0.1, \ldots, 1\}$. Recall that this corresponds to a strategy in which we branch on connections for the the fraction $\rho$ of arcs with the shortest travel time. For the other connections, we branch on arcs.

In Table 1 we have reported solution times for all combinations of separation and branching strategies. These numbers are aggregated values obtained by computing the average over the forty instances.

We see that strategies P2T and P2C are the best performers for most choices of $\rho$. Setting $\rho \neq 0$ yields a positive effect for all separation strategies, although the exact value of $\rho$ does not seem to be that important. In the remainder of the experiments, we will use the combination of $\rho=0.6$ and P2C, as this combination yields the lowest average solution time on our test set. If we compare the solution time of the combination of $\rho=0.6$ and $\mathrm{P} 2 \mathrm{C}$ to the solution time of the combination of $\mathrm{N}$ and $\rho=0$, we see that introducing the precedence inequalities and the branching strategy together yields a factor 6.6 improvement in solution time.

In Table 2, results of using the different separation strategies are presented for $\rho=0.6$. The rows respectively present the average solution time in seconds, the average number of visited nodes of the search trees, the average number of precedence inequalities and 


\begin{tabular}{lrrrrrr}
\hline \multirow{2}{*}{$\begin{array}{l}\text { Seconds } \\
\rho\end{array}$} & \multicolumn{6}{c}{ Separation strategy } \\
\cline { 2 - 7 } & \multicolumn{1}{c}{$\mathrm{N}$} & \multicolumn{1}{c}{$\mathrm{P}$} & $\mathrm{P} 2 \mathrm{~T}$ & $\mathrm{P} 2 \mathrm{C}$ & \multicolumn{1}{c}{$\mathrm{T}$} & $\mathrm{T} 2 \mathrm{C}$ \\
\hline 0.0 & 31.8 & 27.2 & 20.2 & 18.6 & 33.9 & 36.2 \\
0.1 & 13.7 & 10.4 & 7.1 & 7.6 & 8.2 & 8.7 \\
0.2 & 13.5 & 5.7 & 9.3 & 5.4 & 9.3 & 7.3 \\
0.3 & 13.2 & 6.8 & 6.5 & 5.5 & 7.5 & 8.8 \\
0.4 & 12.0 & 10.1 & 6.3 & 6.9 & 8.4 & 8.0 \\
0.5 & 11.1 & 7.2 & 5.7 & 6.5 & 8.7 & 7.8 \\
0.6 & 18.0 & 9.3 & 8.8 & 4.8 & 7.6 & 7.0 \\
0.7 & 10.4 & 8.5 & 7.9 & 6.8 & 10.2 & 7.8 \\
0.8 & 21.4 & 9.8 & 9.9 & 7.1 & 9.9 & 10.6 \\
0.9 & 12.3 & 9.5 & 8.7 & 5.3 & 8.6 & 6.0 \\
1.0 & 22.5 & 11.9 & 9.6 & 6.2 & 9.0 & 5.4 \\
\hline
\end{tabular}

Table 1: Average solution times for various strategies.

rounded capacity inequalities added, and the percentages of the total solution time used for the separation of the precedence inequalities and for the rounded capacity inequalities. The last row displays for how many out of the forty instances the used separation strategy yielded the shortest computation time. If multiple strategies have the same solution time (rounded to milliseconds), they are all counted as best strategies.

\begin{tabular}{rrrrrrr}
\hline & $\mathrm{N}$ & $\mathrm{P}$ & $\mathrm{P} 2 \mathrm{~T}$ & $\mathrm{P} 2 \mathrm{C}$ & $\mathrm{T}$ & $\mathrm{T} 2 \mathrm{C}$ \\
\hline Seconds & 18.0 & 9.3 & 8.8 & 4.8 & 7.6 & 7.0 \\
Nodes & 2,481 & 1,406 & 1,144 & 537 & 848 & 696 \\
Precedence inequalities & 0 & 117 & 107 & 66 & 97 & 101 \\
Rounded capacity inequalities & 575 & 426 & 413 & 341 & 405 & 390 \\
\% of time sep. prec. & $0.0 \%$ & $5.4 \%$ & $6.2 \%$ & $4.4 \%$ & $8.0 \%$ & $8.0 \%$ \\
\% of time sep. cap. & $2.7 \%$ & $3.3 \%$ & $3.0 \%$ & $2.7 \%$ & $3.0 \%$ & $2.6 \%$ \\
Best strategy & $6 / 40$ & $9 / 40$ & $9 / 40$ & $14 / 40$ & $7 / 40$ & $14 / 40$ \\
\hline
\end{tabular}

Table 2: Average branch-and-cut statistics for various separation strategies $(\rho=0.6)$.

If we look at the total solution time, it becomes clear that all strategies yield an improvement over strategy N. Based on the tested instances, strategy P2C yields the lowest computation time; a factor 3.8 better than strategy $\mathrm{N}$.

Looking at the number of nodes in the search trees, we see that all strategies allow the number of nodes to be greatly reduced compared to strategy $\mathrm{N}$. We see that, on average, using $\mathrm{T} 2 \mathrm{C}$ allows for the largest number of violated precedence inequalities to be found per node in the search tree. Still, strategy P2C is more effective. This observation cannot be explained by the increase in separation time alone; the average time spend per instance on separating precedence inequalities is 0.2 seconds for $\mathrm{P} 2 \mathrm{C}$, and 0.6 seconds for $\mathrm{T} 2 \mathrm{C}$.

There are two other effects that may explain the difference between $\mathrm{P} 2 \mathrm{C}$ and $\mathrm{T} 2 \mathrm{C}$. First, as more precedence inequalities are found, larger LP relaxations have to be solved, which takes more time. Second, as more precedence inequalities can be found, it can 
happen that the LP relaxation is resolved more often. It seems that this additional work does not add much value over $\mathrm{P} 2 \mathrm{C}$.

Table 2 shows that P2C is the best strategy for 14 of the test-instances. For T2C, this number is the same. Looking at the disaggregated data (Table 5 in Appendix $\mathrm{C}$ ) we see that for the instances with 20 clients, T2C is the best strategy 5 out of 10 times, while $\mathrm{P} 2 \mathrm{C}$ is never the best strategy. For the instances with 25 clients, however, $\mathrm{P} 2 \mathrm{C}$ is the best strategy 5 out of 10 times, while T2C is the best only once.

Surprisingly, after processing only the root node, there is almost no difference in lower bounds between strategy $\mathrm{N}$ and the other strategies. For 35 instances the lower bounds are exactly the same. For the other instances, differences between lower bounds of at most $0.04 \%$ are observed. The power of the precedence inequalities really shows further down in the search tree. This could be explained by the nature of the precedence inequalities: they disallow certain combinations of paths. If a solution is very fractional, not many paths can be detected, and hence the precedence inequalities are of little use. Deeper in the tree, where more variables are fixed, they become more effective.

\subsection{Comparison with branch-price-and-cut}

Next, we compare the performance of our branch-and-cut algorithm, using strategy P2C and $\rho=0.6$, to the performance of the branch-price-and-cut algorithm in Spliet and Gabor (2014).

To this end, we run their implementation on the same computer as on which our algorithm is run. The computation times are thus directly comparable. The results we present are based on their branch-price-and-cut algorithm with 2-cycle elimination and adding rounded capacity cuts, which is the solution method in Spliet and Gabor (2014) yielding the best average time performance on the test set.

The results per instance can be found in Table 3. Per instance, data is provided on five different categories, both for the branch-price-and-cut algorithm (BP\&C) and the branchand-cut algorithm (B\&C). The columns labeled 'Seconds' indicate the times in seconds for solving the instance to optimality, the maximum time allowed being one hour. The columns 'Nodes' state the number of nodes of the search tree that have been explored during that time. When the algorithm terminates, the percentual deviation from the optimum is given in the column 'Optimality gap'. A value of zero indicates the problem was solved to optimality. The column 'Root gap' shows a similar value, indicating the optimality gap after processing only the root node. The optimality gap and the root gap are calculated ex-post, using the actual optimal value. Finally, the column 'Value' gives the optimal objective value for that instance. 


\begin{tabular}{|c|c|c|c|c|c|c|c|c|c|c|}
\hline \multirow[b]{2}{*}{ Inst. } & \multirow[b]{2}{*}{ Clients } & \multicolumn{2}{|c|}{ Seconds } & \multicolumn{2}{|c|}{ Nodes } & \multicolumn{2}{|c|}{ Optimality gap } & \multicolumn{2}{|c|}{ Root gap } & \multirow{2}{*}{$\frac{\text { Value }}{\mathrm{B} \& \mathrm{C}}$} \\
\hline & & $\mathrm{BP} \& \mathrm{C}$ & $\mathrm{B} \& \mathrm{C}$ & $\mathrm{BP} \& \mathrm{C}$ & $\mathrm{B} \& \mathrm{C}$ & $\mathrm{BP} \& \mathrm{C}$ & $\mathrm{B} \& \mathrm{C}$ & $\mathrm{BP} \& \mathrm{C}$ & $\mathrm{B} \& \mathrm{C}$ & \\
\hline 1 & 10 & 0.7 & 0.0 & 1 & 1 & 0 & 0 & 0 & 0 & 17.65 \\
\hline 2 & 10 & 121.9 & 0.1 & 483 & 18 & 0 & 0 & 0.17 & 0.28 & 15.56 \\
\hline 3 & 10 & 3.7 & 0.0 & 1 & 1 & 0 & 0 & 0 & 0 & 17.42 \\
\hline 4 & 10 & 28.5 & 0.1 & 193 & 6 & 0 & 0 & 0.14 & 0.14 & 18.51 \\
\hline 5 & 10 & 2.3 & 0.3 & 2 & 42 & 0 & 0 & 0 & 0.34 & 16.07 \\
\hline 6 & 10 & 1.5 & 0.0 & 2 & 1 & 0 & 0 & 0 & 0 & 18.00 \\
\hline 7 & 10 & 4.9 & 0.0 & 4 & 1 & 0 & 0 & 0 & 0 & 17.02 \\
\hline 8 & 10 & 3.5 & 0.1 & 29 & 21 & 0 & 0 & 0.65 & 0.96 & 23.89 \\
\hline 9 & 10 & 3.0 & 0.0 & 7 & 1 & 0 & 0 & 0 & 0 & 20.31 \\
\hline 10 & 10 & 5.9 & 0.0 & 5 & 1 & 0 & 0 & 0 & 0 & 16.31 \\
\hline 11 & 15 & 87.4 & 0.1 & 22 & 1 & 0 & 0 & 0 & 0 & 17.78 \\
\hline 12 & 15 & $3,600.0$ & 39.1 & 889 & 14,037 & 0.15 & 0 & 0.67 & 2.36 & 27.10 \\
\hline 13 & 15 & $3,600.0$ & 2.6 & 684 & 587 & 0.59 & 0 & 1.10 & 1.78 & 29.37 \\
\hline 14 & 15 & 58.0 & 0.2 & 45 & 1 & 0 & 0 & 0 & 0.03 & 23.18 \\
\hline 15 & 15 & 29.4 & 0.6 & 36 & 11 & 0 & 0 & 0 & 0.17 & 24.15 \\
\hline 16 & 15 & 92.4 & 0.3 & 98 & 1 & 0 & 0 & 0.10 & 0.17 & 21.03 \\
\hline 17 & 15 & 22.9 & 0.1 & 15 & 1 & 0 & 0 & 0 & 0 & 22.04 \\
\hline 18 & 15 & 105.3 & 0.8 & 98 & 124 & 0 & 0 & 0.20 & 0.47 & 22.30 \\
\hline 19 & 15 & 133.3 & 1.3 & 133 & 210 & 0 & 0 & 0.56 & 0.97 & 26.52 \\
\hline 20 & 15 & 41.6 & 0.4 & 28 & 11 & 0 & 0 & 0 & 0 & 22.11 \\
\hline 21 & 20 & $3,600.0$ & 1.2 & 864 & 48 & 0.02 & 0 & 0.57 & 1.11 & 28.08 \\
\hline 22 & 20 & 152.3 & 9.0 & 62 & 658 & 0 & 0 & 0.03 & 0.19 & 29.80 \\
\hline 23 & 20 & 99.6 & 0.4 & 40 & 1 & 0 & 0 & 0 & 0.12 & 30.30 \\
\hline 24 & 20 & 112.2 & 1.7 & 27 & 58 & 0 & 0 & 0.03 & 0.86 & 24.16 \\
\hline 25 & 20 & $3,600.0$ & 6.9 & 712 & 389 & 0.08 & 0 & 0.61 & 1.09 & 29.84 \\
\hline 26 & 20 & 65.4 & 0.2 & 16 & 1 & 0 & 0 & 0 & 0 & 29.72 \\
\hline 27 & 20 & 85.5 & 0.3 & 24 & 1 & 0 & 0 & 0 & 0 & 26.48 \\
\hline 28 & 20 & 106.5 & 1.1 & 36 & 11 & 0 & 0 & 0 & 0.08 & 26.14 \\
\hline 29 & 20 & 65.5 & 0.5 & 17 & 1 & 0 & 0 & 0 & 0.05 & 26.61 \\
\hline 30 & 20 & 45.1 & 0.3 & 4 & 1 & 0 & 0 & 0 & 0 & 26.36 \\
\hline 31 & 25 & 610.9 & 2.3 & 121 & 20 & 0 & 0 & 0.13 & 0.57 & 31.43 \\
\hline 32 & 25 & 840.0 & 1.3 & 164 & 4 & 0 & 0 & 0.07 & 0 & 30.71 \\
\hline 33 & 25 & $3,600.0$ & 9.4 & 413 & 395 & 0.33 & 0 & 0.45 & 1.03 & 33.71 \\
\hline 34 & 25 & 193.2 & 11.1 & 36 & 391 & 0 & 0 & 0 & 0.33 & 33.34 \\
\hline 35 & 25 & 640.0 & 6.1 & 119 & 201 & 0 & 0 & 0 & 0.85 & 29.05 \\
\hline 36 & 25 & $3,600.0$ & 39.3 & 1,662 & 1,733 & 0.13 & 0 & 0.43 & 1.49 & 30.50 \\
\hline 37 & 25 & $3,600.0$ & 22.4 & 278 & 1,472 & 0.29 & 0 & 0.29 & 0.43 & 28.68 \\
\hline 38 & 25 & $3,600.0$ & 9.7 & 1,259 & 337 & 0.12 & 0 & 0.30 & 0.59 & 35.69 \\
\hline 39 & 25 & $3,600.0$ & 7.2 & 2,294 & 219 & 0 & 0 & 0.50 & 0.94 & 32.55 \\
\hline 40 & 25 & $1,093.2$ & 15.2 & 521 & 471 & 0 & 0 & 0.30 & 0.62 & 32.14 \\
\hline \multicolumn{2}{|c|}{ Average } & 931.4 & 4.8 & 286.1 & 537.2 & 0.04 & 0 & 0.18 & 0.45 & 25.29 \\
\hline
\end{tabular}

Table 3: Comparison of branch-price-and-cut and branch-and-cut. 
What immediately stands out is the enormous decrease in computation time of our new algorithm with respect to the previous algorithm. The instances that can be solved to optimality by the branch-price-and-cut algorithm, can be solved to optimality by the branch-and-cut algorithm 89.6 times faster on average. In total, 9 instances cannot be solved to optimality by the branch-price-and-cut algorithm after a total of 9 hours of computation time. With the branch-and-cut algorithm, all these instances can be solved to optimality in 137.9 seconds. Hence, this is a speedup of at least a factor 234.9.

The new algorithm is faster for all tested instances by at least a factor 7.6 and even up to a factor 2997 for instance 21. If we consider the time necessary to attempt to solve all instances combined, the total time decreases from $37,255.3$ seconds in total to 192.1 seconds; a speedup factor of 193.9. We point out that this difference is not the result of using rounded capacity cuts, as both the branch-and-cut algorithm and the branch-price-and-cut algorithm use these valid inequalities.

It can be seen that an advantage of the branch-price-and-cut algorithm is the stronger LP bound it provides, as for almost all instances the root gap is smaller than the gap given by the branch-and-cut algorithm. However, this strength is less apparent in the remainder of the search tree, as on average the branch-and-cut algorithm processes only twice the number of nodes in the branch-price-and-cut algorithm. There are some extreme instances, however, where processing a lot of nodes is necessary, e.g., instances 12 and 36 . Here, we observe that the branch-and-cut algorithm is able to process a large number of nodes in little time $(14000+$ in less than 40 seconds for instance 12). The branch-priceand-cut algorithm generates stronger bounds, but cannot process enough nodes in the given time to solve the problem.

\subsection{Performance on larger instances}

As our branch-and-cut algorithm is able to solve all 40 test-instances used in Spliet and Gabor (2014), we also test our algorithm on larger instances, as introduced in Section 5.1. Recall that these instances are generated in a similar way as the first forty instances. The only difference is that the number of clients is larger: between 30 and 50 .

In Table 4 we report results for the instances with 30 and 35 clients. This table is structured in the same way as Table 3. The column 'Value' is replaced by the columns 'Lower bound' and 'Upper bound', as not all instances can be solved to optimality. The reported root gaps are no longer ex post, but based on the best known upper bound.

It can be seen that all but one of the instances with 30 clients can be solved to optimality within one hour of computation time, and for the remaining instance we find a solution with an optimality gap of $1.66 \%$.

The instances with 35 clients are more difficult: 6 out of the 10 instances can be solved to optimality within one hour. The remaining instances have an optimality gap of less than $1.32 \%$.

The instances with 40, 45 and 50 customers cannot be solved consistently by our branch-and-cut algorithm, which proves optimality of the found solution for only two of these instances in one hour of computation time. In Table 6 in Appendix C, we report the results for these instances, including best found lower and upper bounds. The instances with 40 clients have optimality gaps below 2.09\%. The instances with 45 clients have optimality gaps between $0.48 \%$ and $7.12 \%$. The instances with 50 clients have optimality gaps between $3.09 \%$ and $7.23 \%$. 


\begin{tabular}{rrrrrrrr}
\hline Inst. & Clients & Seconds & Nodes & Optimality gap & Root gap & Lower bound & Upper bound \\
\hline 41 & 30 & 137.0 & 3,281 & 0 & 1.99 & 36.38 & 36.38 \\
42 & 30 & $3,600.0$ & 40,701 & 1.66 & 3.48 & 34.16 & 34.74 \\
43 & 30 & 187.6 & 8,317 & 0 & 1.91 & 35.48 & 35.48 \\
44 & 30 & 60.6 & 1,296 & 0 & 1.46 & 35.88 & 35.88 \\
45 & 30 & 110.8 & 4,158 & 0 & 1.78 & 35.55 & 35.55 \\
46 & 30 & 7.7 & 165 & 0 & 0.58 & 37.47 & 37.47 \\
47 & 30 & 17.8 & 292 & 0 & 0.70 & 32.54 & 32.54 \\
48 & 30 & 357.9 & 9,657 & 0 & 1.84 & 36.32 & 36.32 \\
49 & 30 & 930.3 & 24,789 & 0 & 2.34 & 35.30 & 35.30 \\
50 & 30 & 30.7 & 710 & 0 & 0.72 & 40.27 & 40.27 \\
\hline 51 & 35 & 18.2 & 114 & 0 & 0.78 & 43.46 & 43.46 \\
52 & 35 & 14.0 & 69 & 0 & 0.06 & 41.84 & 41.84 \\
53 & 35 & $3,600.0$ & 32,201 & 1.32 & 2.86 & 44.54 & 45.14 \\
54 & 35 & $3,600.0$ & 50,601 & 0.87 & 3.06 & 41.20 & 41.57 \\
55 & 35 & 68.5 & 983 & 0 & 0.68 & 37.92 & 37.92 \\
56 & 35 & $3,600.0$ & 42,289 & 0.92 & 2.58 & 44.08 & 44.49 \\
57 & 35 & $3,600.0$ & 31,201 & 0.44 & 1.85 & 40.65 & 40.83 \\
58 & 35 & 127.9 & 1,871 & 0 & 0.89 & 41.22 & 41.22 \\
59 & 35 & 245.1 & 2,485 & 0 & 0.93 & 43.43 & 43.43 \\
60 & 35 & 443.3 & 6,655 & 0 & 1.32 & 42.27 & 42.27 \\
\hline
\end{tabular}

Table 4: Result for the branch-and-cut algorithm on instances 41 to 60 . 


\section{Conclusion}

In this paper we present a compact formulation for the TWAVRP based on the 2commodity flow formulation introduced by Baldacci et al. (2004) and the MTZ-inequalities introduced by Miller et al. (1960). We use this formulation in a branch-and-cut algorithm in which rounded capacity cuts are separated in each node of the search tree.

To further improve the performance of our algorithm, we introduce a branching rule and a novel class of valid inequalities: the precedence inequalities. These TWAVRP specific inequalities are hard to separate in general. Therefore, we introduce exact separation algorithms for two subsets, the path precedence inequalities and the tournament precedence inequalities. Furthermore, we extend these algorithms to separation heuristics for general precedence inequalities. Using the branching rule and separating precedence inequalities makes our algorithm 6.6 times faster.

The new algorithm is superior to the best known algorithm in the literature, the algorithm of Spliet and Gabor (2014), for all tested instances. Overall, an average speedup factor of 193.9 is achieved.

Finally, we test our algorithm on larger instances. Of the instances with 30 clients, 9 out of 10 instances could be solved to optimality within the one hour time limit. For the instances with 35 clients, we found the optimal solution for 6 out of 10 instances. The instances that could not be solved to optimality all have an optimality gap of less than $1.66 \%$. Instances with 40 clients and more, however, could not be solved to optimality consistently.

In this paper, we compare our results to the branch-price-and-cut algorithm of Spliet and Gabor (2014). Even without the use of precedence inequalities, our algorithm shows a substantial speedup over the branch-price-and-cut algorithm. It it still interesting, though, to investigate the effect of incorporating the precedence inequalities in a branchprice-and-cut algorithm. Similarly, it is interesting to see how the precedence inequalities would perform on the DTWAVRP.

Another interesting topic of further research concerns algorithms for separating precedence inequalities. In the future, TWAVRP algorithms would benefit from new algorithms for separating the remaining class of precedence inequalities, corresponding to directed cyclic graphs. 


\section{References}

Norbert Ascheuer, Matteo Fischetti, and Martin Grötschel. A Polyhedral Study of the Asymmetric Traveling Salesman Problem with Rime Qindows. Networks, 36(2):69-79, 2000 .

Norbert Ascheuer, Matteo Fischetti, and Martin Grötschel. Solving the Asymmetric Travelling Salesman Problem with time windows by branch-and-cut. Mathematical Programming, 90(3):475-506, 2001.

Roberto Baldacci, Eleni Hadjiconstantinou, and Aristide Mingozzi. An Exact Algorithm for the Capacitated Vehicle Routing Problem Based on a Two-Commodity Network Flow Formulation. Operations Research, 52(5):723-738, 2004.

Roberto Baldacci, Aristide Mingozzi, and Roberto Roberti. Recent exact algorithms for solving the vehicle routing problem under capacity and time window constraints. European Journal of Operational Research, 218(1):1-6, 2012.

Michel L Balinski and Richard E Quandt. On an Integer Program for a Delivery Problem. Operations Research, 12(2):300-304, 1964.

Guy Desaulniers, François Lessard, and Ahmed Hadjar. Tabu Search, Partial Elementarity, and Generalized k-path Inequalities for the Vehicle Routing Problem with Time Windows. Transportation Science, 42(3):387-404, 2008.

Chris Groër, Bruce Golden, and Edward Wasil. The Consistent Vehicle Routing Problem. Manufacturing $\&$ service operations management, 11(4):630-643, 2009.

Ola Jabali, Roel Leus, Tom Van Woensel, and Ton De Kok. Self-imposed time windows in vehicle routing problems. OR Spectrum, 37(2):331-352, 2015.

Arthur B Kahn. Topological Sorting of Large Networks. Communications of the ACM, 5(11):558-562, 1962.

Attila A Kovacs, Bruce L Golden, Richard F Hartl, and Sophie N Parragh. Vehicle Routing Problems in Which Consistency Considerations are Important: A Survey. Networks, 64(3):192-213, 2014.

Gilbert Laporte, Yves Nobert, and Martin Desrochers. Optimal Routing under Capacity and Distance Restrictions. Operations research, 33(5):1050-1073, 1985.

Jens Lysgaard. CVRPSEP: A package of separation routines for the Capacitated Vehicle Routing Problem. Working paper, Aarhus School of Business, 2003.

Jens Lysgaard, Adam N Letchford, and Richard W Eglese. A new branch-and-cut algorithm for the capacitated vehicle routing problem. Mathematical Programming, 100 (2):423-445, 2004.

Clair E Miller, Albert W Tucker, and Richard A Zemlin. Integer Programming Formulation of Traveling Salesman Problems. Journal of the ACM (JACM), 7(4):326-329, 1960 . 
Christos H Papadimitriou. The Euclidean travelling salesman problem is NP-complete. Theoretical Computer Science, 4(3):237-244, 1977.

Remy Spliet and Guy Desaulniers. The discrete time window assignment vehicle routing problem. European Journal of Operational Research, 244(2):379-391, 2015.

Remy Spliet and Adriana F Gabor. The Time Window Assignment Vehicle Routing Problem. Transportation Science, 49(4):721-731, 2014. 


\section{A Proof Proposition 1}

Proposition 1. All integer feasible solutions to (1)-(14), (16), (17), (19), and (20)-(23) satisfy

$$
x_{i j}^{\omega} \in \mathbb{B} \forall(i, j) \in A, \omega \in \Omega .
$$

Proof. Constraints (20) and (21) state that $x_{0 j}^{\omega}, x_{j, n+1}^{\omega} \in \mathbb{B}$ for all $j \in V^{\prime}$. Next, we will prove that if $x_{i j}^{\omega}=1$ with $i \in V^{\prime} \cup\{0\}$ and $j \in V^{\prime}$, then there exists a unique $k \in V^{\prime} \cup\{n+1\}, k \neq i$ such that $x_{j k}^{\omega}=1$.

Suppose that $x_{i j}^{\omega}=1$ for some $i \in V^{\prime} \cup\{0\}$ and $j \in V^{\prime}$. Due to the flow conservation constraints $x_{l j}^{\omega}=0$ for all $l \neq i$ and furthermore there exists a vertex $k \in V^{\prime} \cup\{n+1\}$ such that $x_{j k}^{\omega}>0$. If $k=n+1$, then by integrality of the flows going into the depot, we have $x_{j k}^{\omega}=1$ and by the flow conservation constraints we have $x_{j l}^{\omega}=0$ for all $l \neq k$. Finally, suppose $k \neq n+1$. Note that $k \neq i$ as $x_{j k}>0$ while $x_{j i}=0$ because $x_{i j}+x_{j i}=1$. Constraints (22) and (23) state that $x_{j k}^{\omega}+x_{k j}^{\omega}=1$ and since $x_{l j}^{\omega}=0$ for all $l \neq i$, it follows that $x_{j k}^{\omega}=1$. Using again the flow conservation constraints, $x_{j l}=0$ for all $l \neq k$.

We know that all flows out of the depot are equal to one. We have just proven that any vertex in $V^{\prime}$ with a single inflow of 1 also has a single outflow of 1 . It follows that all flows between the depots are of size 1 .

What remains to be proven is that all clients are contained in the integral flows between the depots. Because of the flow conservation constraints (2)-(3), the only other possibility is that there exists a cycle, given by edges $(1,2),(2,3), \ldots,(k-1, k),(k, 1)$ such that for each arc $(i, j)$ in this cycle we have $x_{i j}^{\omega}+x_{j i}^{\omega}=1$. Using the flow conservation constraints (2)-(3), we have that all arcs adjacent to this cycle have zero flow. Constraints (4) and (16) together state that if $x_{i j}+x_{j i}=0$, then $z_{i j}=z_{j i}=0$. Hence, if a cycle exists, (5) contains the following constraints:

$$
\begin{aligned}
z_{k, 1}^{\omega}-z_{1, k}^{\omega}+z_{2,1}^{\omega}-z_{1,2}^{\omega} & =2 d_{1}^{\omega} \\
z_{1,2}^{\omega}-z_{2,1}^{\omega}+z_{3,2}^{\omega}-z_{2,3}^{\omega} & =2 d_{2}^{\omega} \\
& \vdots \\
z_{k-1, k}^{\omega}-z_{k, k-1}^{\omega}+z_{1, k}^{\omega}-z_{k, 1}^{\omega} & =2 d_{k}^{\omega}
\end{aligned}
$$

Summing these constraints gives $0=2 \sum_{i=1}^{k} d_{i}^{\omega}>0$, which is a contradiction. Hence, all clients are contained in the integer flows between the depots. It follows that all $x$-variables are integer and that (15) is satisfied. 


\section{B Separating precedence inequalities is co-NP-hard}

We prove that separating precedence inequalities is co-NP-hard. First, we present a brief outline of the proof.

We construct a specific instance of the TWAVRP and a corresponding optimal solution to the LP relaxation of (1)-(16), (17) and (19). This optimal solution to the LP relaxation is an instance of the separation problem of finding violated precedence inequalities. We will refer to this instance as instance $I$. Next, we characterize this instance of the separation problem as a decision problem. Finally, we show that separating precedence inequalities is co-NP-hard. We show this by a polynomial time reduction from Euclidean TSP.

\section{TWAVRP instance}

Consider $n \geq 4$ clients, travel times $t_{i j}$ adhering to the triangle inequality, and endogenous time window widths $w_{i}$. Let $t^{\max }$ be the maximum of the given travel times, and let $w^{\max }$ be the maximum of the given endogenous time window widths.

We set $s_{i}=0$ and $e_{i}=2 t^{\max }+w^{\max }$ for all locations $i \in V$ and we define two scenarios $\Omega=\{1,2\}$, both with probability 0.5 of occurring. In both scenarios, we set the demand of every client equal to 1 . The vehicle capacity $Q$ is set equal to $n$. We set all travel costs equal to zero, such that any feasible solution to the LP relaxation is also an optimal solution.

\section{Solution to the LP relaxation}

Next, we describe an optimal solution to the LP relaxation. We start with setting the $x$-variables. For scenario 1 , set $x_{1 i}^{1}=x_{i j}^{1}=x_{i n}^{1}=\frac{1}{n-2}$ for all $i=2,3, \ldots, n-1$ and $j=2,3, \ldots, n-1(i \neq j)$ and set $x_{01}^{1}=x_{n, n+1}^{1}=1$. All other $x$-variables for scenario 1 are set to zero.

For scenario 2, we set $x_{01}^{2}=x_{n, n+1}^{2}=1-\frac{1}{n-1}$. Furthermore, we set $x_{0 i}^{2}=x_{i, n+1}^{2}=1$ for all $i=2,3, \ldots n-1$. Moreover, we set $x_{0 n}^{2}=x_{1, n+1}^{2}=1$ and $x_{n 1}^{2}=\frac{1}{n-1}$. The remaining flow variables are set to zero.

As an example, Figures 2 and 3 present the flows given by the $x$-variables when $n=4$.

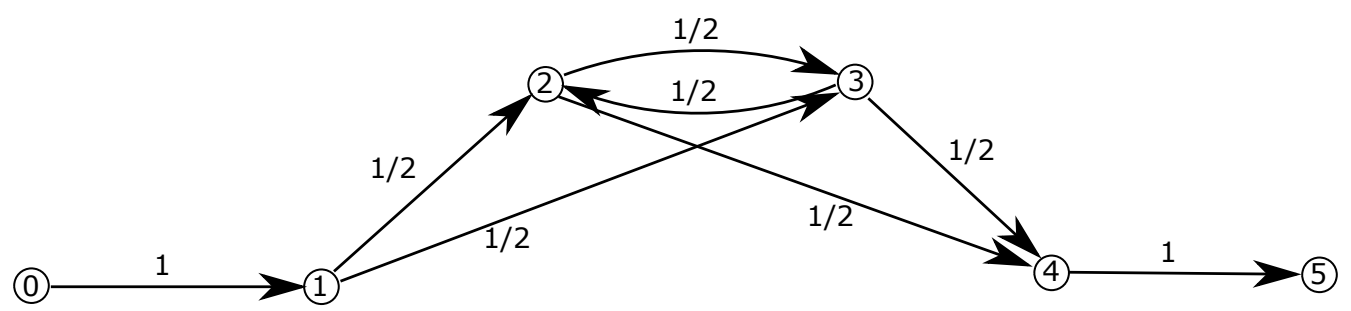

Figure 2: Flows given by the $x$-variables in scenario 1 when $n=4$.

Next, we also present an assignment for the $z$-variables. For scenario 1 we set $z_{01}^{1}=$ $z_{n+1, n}^{1}=n$. Furthermore, we set $z_{1 i}^{1}=z_{n i}^{1}=\frac{n-1}{n-2}$ and $z_{i 1}^{1}=z_{i n}^{1}=\frac{1}{n-2}$ for all $i=$ $2,3, \ldots, n-1$. Finally, we set $z_{i j}^{1}=\frac{n}{n-2}$ for all $i=2,3, \ldots, n-1$ and $j=2,3, \ldots, n-1$ $(i \neq j)$. All other $z$-variables are set to zero.

For scenario 2 we set $z_{01}^{2}=\frac{n-2}{n-1}, z_{10}^{2}=n-2, z_{0 n}^{2}=\frac{n}{n-1}, z_{n 0}^{2}=n-\frac{n}{n-1}, z_{1 n}^{2}=1$, $z_{n 1}^{2}=\frac{1}{n-1}, z_{n+1,1}^{2}=n, z_{n+1, n}^{2}=\frac{n(n-2)}{n-1}$ and finally $z_{0 i}^{2}=1, z_{i 0}^{2}=n-1$ and $z_{n+1, i}^{2}=n$ for all $i=2,3, \ldots, n-1$. All remaining $z$-variables are set to zero.

Finally, set $y_{i}=t^{\max }$ for all $i \in V^{\prime}$ and $t_{i}^{\omega}=t^{\max }$ for all $i \in V^{\prime}$ and all $\omega \in \Omega$. 


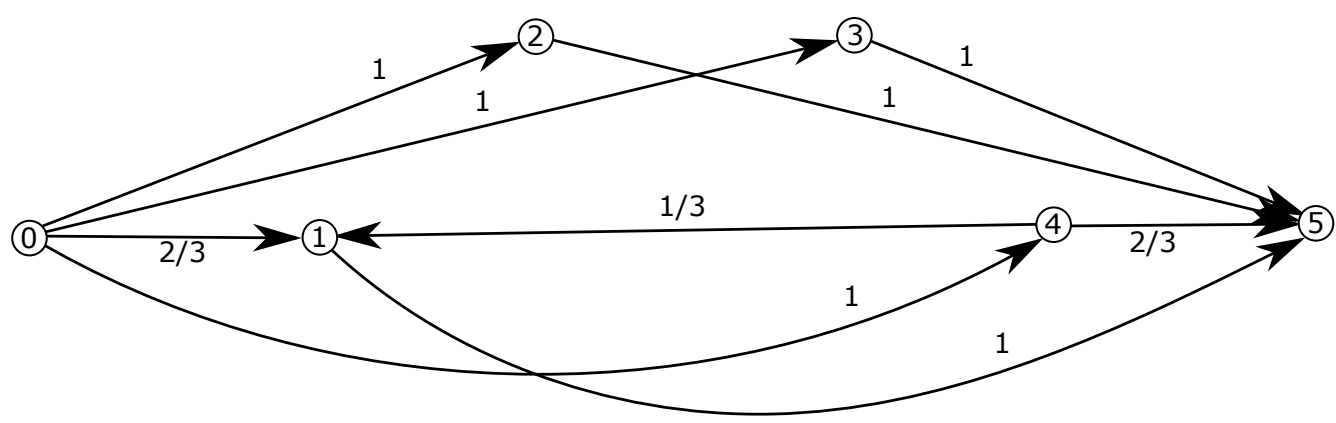

Figure 3: Flows given by the $x$-variables in scenario 2 when $n=4$.

It is straightforward to check that the described variables give a feasible solution to the LP relaxation of (1)-(16), (17) and (19). As all costs are equal to zero, this solution is also optimal. By definition, our optimal solution to the LP relaxation is an instance of the separation problem. In the remainder, we refer to this instance of the separation problem as instance $I$.

\section{Characterization as a decision problem}

Next, we characterize instance $I$ of the separation problem as a decision problem. First, we prove the following lemma.

Lemma 14. If instance I contains a violated precedence inequality, then the only violated precedence inequality is given by (using the notation of Theorem 4) $i=1, j=n, S=$ $\{2,3, \ldots, n-1\}, F=(1: S) \cup(S: S) \cup(S: n), S^{\prime}=\emptyset$ and $F^{\prime}=\{(n, 1)\}$.

Proof. By definition, $F^{\prime}$ cannot contain arcs involving the depot vertices. Therefore, considering the assigned values of the $x$-variables in scenario 2 , we have that the arc $(n, 1)$ is the only arc with non-zero flow that may be in $F^{\prime}$. Hence, $\sum_{(k, l) \in F^{\prime}} x_{k l}^{2}=0$ if $(n, 1) \notin F^{\prime}$ or $0<\sum_{(k, l) \in F^{\prime}} x_{k l}^{2}=\frac{1}{n-1}<1$ if $(n, 1) \in F^{\prime}$. By Lemma 5 we have that $\sum_{(k, l) \in F^{\prime}} x_{k l}^{2}>\left|S^{\prime}\right| \geq 0$. Thus, $(n, 1)$ is contained in $F^{\prime}$. Using that $\sum_{(k, l) \in F^{\prime}} x_{k l}^{2}<1$ and $\sum_{(k, l) \in F^{\prime}} x_{k l}^{2}>\left|S^{\prime}\right|$ it follows that $\left|S^{\prime}\right|=0$ and hence $S^{\prime}=\emptyset$. As $S^{\prime}=\emptyset$ and $(n, 1) \in F^{\prime}$, it follows that $i=1, j=n$ and $F^{\prime}=\{(n, 1)\}$. It remains to be shown that $S=\{2,3, \ldots, n-1\}$ and $F=(1: S) \cup(S: S) \cup(S: n)$.

Suppose by contradiction that $S \in \mathcal{S}(1, n)$ is such that $|S| \leq n-3$. In this case, the largest set $F \in \mathcal{F}(1, S, n)$ is given by $F=(1: S) \cup(S: S) \cup(S: n)$. Therefore, the number of arcs in $F$ is bounded by $|1: S|+|S: S|+|S: n|=|S|+|S|(|S|-1)+|S|=|S|(|S|+1)$. For instance $I$, all these arcs have flow equal to $\frac{1}{n-2}$. It follows that $\sum_{(k, l) \in F} x_{k l}^{1} \leq|S|(|S|+$ 1) $\frac{1}{n-2}$ for all $S \in \mathcal{S}(1, n)$ and $F \in \mathcal{F}(1, S, n)$. Recall that $\sum_{(k, l) \in F^{\prime}} x_{k l}^{2}=\frac{1}{n-1}$ and $\left|S^{\prime}\right|=0$. Using $|S|+1 \leq n-2$ we derive $\sum_{(k, l) \in F} x_{k l}^{1}+\sum_{(k, l) \in F^{\prime}} x_{k l}^{2} \leq|S|(|S|+1) \frac{1}{n-2}+\frac{1}{n-1} \leq$ $|S|+\left|S^{\prime}\right|+1$ for all $|S| \leq n-3$. By Theorem 4 , the corresponding precedence inequality is not violated, which is a contradiction. It follows that $|S|=n-2$, which implies $S=\{2,3, \ldots, n-1\}$.

Finally, we show that $F=(1: S) \cup(S: S) \cup(S: n)$ by contradiction. Suppose that $F \neq(1: S) \cup(S: S) \cup(S: n)$. Recall that $S=\{2,3, \ldots, n-1\}$ and thus $|S|=n-2$. Therefore, $|F| \leq|S|(|S|+1)-1=(n-2)(n-1)-1$. Hence, $\sum_{(k, l) \in F} x_{k l}^{1}+\sum_{(k, l) \in F} x_{k l}^{2} \leq$ $((n-2)(n-1)-1) \frac{1}{n-2}+\frac{1}{n-1}=n-1-\frac{1}{n-2}+\frac{1}{n-1} \leq n-1=|S|+\left|S^{\prime}\right|+1$, which is a contradiction of the precedence inequality being violated. It follows that $F=(1$ : $S) \cup(S: S) \cup(S: n)$. 
Hence, the only potentially violated precedence inequality is given by $i=1, j=n$, $S=\{2,3, \ldots, n-1\}, F=(1: S) \cup(S: S) \cup(S: n), S^{\prime}=\emptyset$ and $F^{\prime}=\{(n, 1)\}$. By assumption, a violated precedence inequality exists, hence, this is the only one.

The next corollary follows from Lemma 14 and characterizes instance $I$ of the separation problem as a decision problem.

Corollary 15. Instance I contains a violated precedence inequality if and only if

$$
\delta_{1 n}(S, F)>w_{1}+w_{n}-t_{n 1}
$$

with $S=\{2,3, \ldots, n-1\}$ and $F=(1: S) \cup(S: S) \cup(S: n)$.

Proof. Assume instance $I$ contains a violated precedence inequality. By Lemma 14, $i=1, j=n, S=\{2,3, \ldots, n-1\}, F=(1: S) \cup(S: S) \cup(S: n), S^{\prime}=\emptyset$ and $F^{\prime}=\{(n, 1)\}$. By Theorem 4 all precedence inequalities satisfy $\delta_{1 n}(S, F)+\delta_{n 1}\left(S^{\prime}, F^{\prime}\right)>$ $w_{1}+w_{n}$. Substituting $\delta_{n 1}\left(S^{\prime}, F^{\prime}\right)=\delta_{n 1}(\emptyset,\{(n, 1)\})=t_{n 1}$ then gives $(36)$.

For the reverse implication, let $S=\{2,3, \ldots, n-1\}, F=(1: S) \cup(S: S) \cup(S: n)$, $S^{\prime}=\emptyset$ and $F^{\prime}=\{(n, 1)\}$ and assume that $\delta_{1 n}(S, F)>w_{1}+w_{n}-t_{n 1}$, which is equivalent to $\delta_{1 n}(S, F)+\delta_{n 1}\left(S^{\prime}, F^{\prime}\right)>w_{1}+w_{n}$ as shown earlier. Similar as in the proof of Lemma 14, we have $\sum_{(k, l) \in F} x_{k l}^{1}+\sum_{(k, l) \in F^{\prime}} x_{k l}^{2}=|S|(|S|+1) \frac{1}{n-2}+\frac{1}{n-1}=n-1+\frac{1}{n-1}>|S|+\left|S^{\prime}\right|+1$. Hence, by Theorem 4, we have that $i, j, S, F, S^{\prime}$ and $F^{\prime}$ define a violated precedence inequality.

\section{The decision problem is co-NP-complete}

Next, we show that the decision problem $\delta_{1 n}(S, F)>w_{1}+w_{n}-t_{n 1}$ is co-NP-complete. First, we note that the decision problem is in co-NP. A polynomial certificate for the $N O$-answer is given by a path from 1 to $n$ visiting all vertices of $S$ and using only arcs of $F$, of length $w_{1}+w_{n}-t_{n 1}$ or less. Next, we show that the decision problem is co-NP complete. We show this with a reduction from Euclidean TSP.

Consider the Euclidean TSP problem: given $n$ distinct points in Euclidean space, determine whether there exists a tour visiting all vertices with total travel time $\alpha$ or less. The travel time between two points is given by their Euclidean distance. Let these travel times be $t_{i j}$. Clearly, the travel times are positive and satisfy the triangle inequality.

Next, we show that the Euclidean TSP problem can be reduced to answering a polynomial number of instances of the complement of the decision problem $\delta_{1 n}(S, F)>$ $w_{1}+w_{n}-t_{n 1}$. For every edge $(k, l)$ we do the following. Without loss of generality, relabel the vertices such that $k=1$ and $l=n$. Now construct an instance $I$ of the separation problem by using the same $n$ and $t$, and by arbitrarily choosing $w_{i} \geq 0$ for all $i \in V^{\prime}$ in such a way that $w_{1}+w_{n}=\alpha$. Next, determine whether $\delta_{1 n}(S, F) \leq w_{1}+w_{n}-t_{n 1}$, which is the complement of $\delta_{1 n}(S, F)>w_{1}+w_{n}-t_{n 1}$. If for any $(k, l)$ the answer is $Y E S$, this implies that there exists a tour of length at most $w_{1}+w_{n}-t_{n 1}+t_{n 1}=\alpha$, and hence the answer to the Euclidean TSP problem is $Y E S$. If the answer is $N O$ for all $(k, l)$, it follows that it is impossible to construct a tour that is sufficiently short. Hence, the answer to the Euclidean TSP problem is $N O$.

As the number of edges is polynomial, we solve the Euclidean TSP problem by answering the complement of the decision problem $\delta_{1 n}(S, F)>w_{1}+w_{n}-t_{n 1}$ a polynomial number of times. As Euclidean TSP is NP-complete (Papadimitriou, 1977), it follows that answering $\delta_{1 n}(S, F)>w_{1}+w_{n}-t_{n 1}$ is co-NP-complete. 
Separating precedence inequalities is co-NP-hard

Finally, consider a separation algorithm that returns a violated precedence inequality if one exists, or returns that no violated precedence inequality exists. Now use this separation algorithm on instance $I$. By Corollary 15, there is only one potential precedence inequality in $I$ that may be violated. Hence, if a violated precedence inequality is found, it is established that $\delta_{1 n}(S, F)>w_{1}+w_{n}-t_{n 1}$, for a given $S$ and $F$. Since determining whether $\delta_{1 n}(S, F)>w_{1}+w_{n}-t_{n 1}$ is co-NP-complete, it follows that the problem of separating precedence inequalities is co-NP-hard in general.

\section{Additional tables}




\begin{tabular}{|c|c|c|c|c|c|c|c|}
\hline \multirow[b]{2}{*}{ Inst. } & \multirow[b]{2}{*}{ Clients } & \multicolumn{6}{|c|}{ Seconds } \\
\hline & & $\mathrm{N}$ & $\mathrm{P}$ & $\mathrm{P} 2 \mathrm{~T}$ & $\mathrm{P} 2 \mathrm{C}$ & $\mathrm{T}$ & $\mathrm{T} 2 \mathrm{C}$ \\
\hline 1 & 10 & 0.0 & 0.0 & 0.0 & 0.0 & 0.0 & 0.0 \\
\hline 2 & 10 & 0.6 & 0.1 & 0.1 & 0.1 & 0.1 & 0.1 \\
\hline 3 & 10 & 0.0 & 0.0 & 0.0 & 0.0 & 0.0 & 0.0 \\
\hline 4 & 10 & 0.8 & 0.1 & 0.1 & 0.1 & 0.1 & 0.1 \\
\hline 5 & 10 & 0.2 & 0.1 & 0.1 & 0.3 & 0.1 & 0.1 \\
\hline 6 & 10 & 0.0 & 0.0 & 0.0 & 0.0 & 0.0 & 0.0 \\
\hline 7 & 10 & 0.0 & 0.0 & 0.0 & 0.0 & 0.0 & 0.0 \\
\hline 8 & 10 & 0.4 & 0.1 & 0.1 & 0.1 & 0.2 & 0.1 \\
\hline 9 & 10 & 0.0 & 0.0 & 0.0 & 0.0 & 0.0 & 0.0 \\
\hline 10 & 10 & 0.0 & 0.0 & 0.0 & 0.0 & 0.0 & 0.0 \\
\hline 11 & 15 & 0.1 & 0.1 & 0.1 & 0.1 & 0.1 & 0.1 \\
\hline 12 & 15 & 68.0 & 143.2 & 95.5 & 39.1 & 60.8 & 63.8 \\
\hline 13 & 15 & 8.7 & 3.9 & 3.7 & 2.6 & 4.1 & 3.0 \\
\hline 14 & 15 & 0.2 & 0.2 & 0.2 & 0.2 & 0.7 & 0.7 \\
\hline 15 & 15 & 1.6 & 0.5 & 0.9 & 0.6 & 0.5 & 0.6 \\
\hline 16 & 15 & 0.9 & 0.3 & 0.3 & 0.3 & 0.3 & 0.3 \\
\hline 17 & 15 & 0.1 & 0.1 & 0.1 & 0.1 & 0.1 & 0.1 \\
\hline 18 & 15 & 1.7 & 0.8 & 1.7 & 0.8 & 2.2 & 1.7 \\
\hline 19 & 15 & 0.7 & 0.7 & 0.8 & 1.3 & 1.3 & 1.3 \\
\hline 20 & 15 & 0.2 & 1.2 & 0.7 & 0.4 & 0.7 & 0.4 \\
\hline 21 & 20 & 1.2 & 1.1 & 1.2 & 1.2 & 1.1 & 1.2 \\
\hline 22 & 20 & 8.1 & 1.9 & 5.9 & 9.0 & 5.9 & 1.3 \\
\hline 23 & 20 & 0.3 & 0.4 & 0.4 & 0.4 & 0.4 & 0.5 \\
\hline 24 & 20 & 1.8 & 2.1 & 3.5 & 1.7 & 3.6 & 1.7 \\
\hline 25 & 20 & 13.5 & 12.2 & 7.1 & 6.9 & 8.3 & 4.4 \\
\hline 26 & 20 & 0.3 & 0.3 & 0.3 & 0.2 & 0.3 & 0.2 \\
\hline 27 & 20 & 2.1 & 0.4 & 0.4 & 0.3 & 0.5 & 0.3 \\
\hline 28 & 20 & 1.1 & 1.0 & 1.2 & 1.1 & 1.2 & 1.1 \\
\hline 29 & 20 & 0.6 & 0.5 & 0.5 & 0.5 & 0.5 & 0.5 \\
\hline 30 & 20 & 0.2 & 0.3 & 0.3 & 0.3 & 0.3 & 0.3 \\
\hline 31 & 25 & 12.0 & 15.7 & 18.2 & 2.3 & 5.0 & 15.0 \\
\hline 32 & 25 & 5.5 & 4.3 & 8.0 & 1.3 & 2.8 & 5.3 \\
\hline 33 & 25 & 35.3 & 9.2 & 37.8 & 9.4 & 11.3 & 14.4 \\
\hline 34 & 25 & 2.6 & 8.4 & 2.3 & 11.1 & 2.4 & 15.8 \\
\hline 35 & 25 & 26.6 & 4.6 & 15.1 & 6.1 & 19.0 & 6.2 \\
\hline 36 & 25 & 33.9 & 39.0 & 31.9 & 39.3 & 31.5 & 47.7 \\
\hline 37 & 25 & 339.9 & 42.7 & 70.7 & 22.4 & 75.4 & 23.6 \\
\hline 38 & 25 & 79.4 & 12.9 & 14.5 & 9.7 & 17.7 & 23.2 \\
\hline 39 & 25 & 51.3 & 35.5 & 10.1 & 7.2 & 17.1 & 37.0 \\
\hline 40 & 25 & 20.5 & 27.0 & 18.3 & 15.2 & 27.2 & 6.7 \\
\hline \multicolumn{2}{|c|}{ Average } & 18.0 & 9.3 & 8.8 & 4.8 & 7.6 & 7.0 \\
\hline
\end{tabular}

Table 5: Solution times for various strategies, using $\rho=0.6$. 


\begin{tabular}{|c|c|c|c|c|c|c|c|}
\hline Inst. & Clients & Seconds & Nodes & Optimality gap & Root gap & Lower bound & Upper bound \\
\hline 61 & 40 & $3,600.0$ & 17,282 & 2.09 & 3.29 & 45.38 & 46.35 \\
\hline 62 & 40 & 550.3 & 8,479 & 0 & 1.08 & 48.35 & 48.35 \\
\hline 63 & 40 & $3,600.0$ & 36,554 & 0.23 & 1.83 & 44.38 & 44.48 \\
\hline 64 & 40 & $3,169.7$ & 18,069 & 0 & 1.88 & 43.75 & 43.75 \\
\hline 65 & 40 & $3,600.0$ & 37,915 & 0.77 & 2.22 & 43.13 & 43.46 \\
\hline 66 & 40 & $3,600.0$ & 32,601 & 1.22 & 2.79 & 44.14 & 44.68 \\
\hline 67 & 40 & $3,600.0$ & 27,201 & 0.67 & 1.89 & 46.64 & 46.96 \\
\hline 68 & 40 & $3,600.0$ & 16,210 & 1.76 & 2.88 & 44.23 & 45.02 \\
\hline 69 & 40 & $3,600.0$ & 17,822 & 1.88 & 3.25 & 42.39 & 43.20 \\
\hline 70 & 40 & $3,600.0$ & 16,901 & 0.68 & 1.76 & 42.71 & 43.00 \\
\hline 71 & 45 & $3,600.0$ & 5,741 & 4.36 & 5.08 & 49.52 & 51.78 \\
\hline 72 & 45 & $3,600.0$ & 9,201 & 2.69 & 3.40 & 50.73 & 52.13 \\
\hline 73 & 45 & $3,600.0$ & 14,001 & 0.48 & 1.70 & 41.50 & 41.70 \\
\hline 74 & 45 & $3,600.0$ & 4,789 & 1.23 & 2.18 & 47.25 & 47.84 \\
\hline 75 & 45 & $3,600.0$ & 9,501 & 2.19 & 2.90 & 48.77 & 49.86 \\
\hline 76 & 45 & $3,600.0$ & 6,401 & 7.12 & 7.61 & 48.38 & 52.09 \\
\hline 77 & 45 & $3,600.0$ & 12,064 & 2.13 & 2.87 & 50.09 & 51.18 \\
\hline 78 & 45 & $3,600.0$ & 8,501 & 3.58 & 4.18 & 52.02 & 53.95 \\
\hline 79 & 45 & $3,600.0$ & 20,601 & 1.58 & 2.71 & 47.45 & 48.21 \\
\hline 80 & 45 & $3,600.0$ & 10,097 & 1.99 & 2.75 & 49.57 & 50.57 \\
\hline 81 & 50 & $3,600.0$ & 5,898 & 3.46 & 4.08 & 56.81 & 58.85 \\
\hline 82 & 50 & $3,600.0$ & 5,701 & 3.19 & 3.85 & 51.50 & 53.20 \\
\hline 83 & 50 & $3,600.0$ & 4,001 & 5.31 & 5.61 & 57.45 & 60.67 \\
\hline 84 & 50 & $3,600.0$ & 4,401 & 7.23 & 7.71 & 52.31 & 56.38 \\
\hline 85 & 50 & $3,600.0$ & 3,146 & 4.17 & 4.92 & 53.74 & 56.07 \\
\hline 86 & 50 & $3,600.0$ & 3,585 & 5.63 & 6.14 & 51.68 & 54.76 \\
\hline 87 & 50 & $3,600.0$ & 6,611 & 3.09 & 3.65 & 52.47 & 54.14 \\
\hline 88 & 50 & $3,600.0$ & 6,801 & 3.68 & 4.39 & 54.82 & 56.91 \\
\hline 89 & 50 & $3,600.0$ & 4,001 & 3.71 & 4.06 & 59.23 & 61.51 \\
\hline 90 & 50 & $3,600.0$ & 4,901 & 3.15 & 3.69 & 57.68 & 59.55 \\
\hline
\end{tabular}

Table 6: Results for the branch-and-cut algorithm on instances 61 to 90. 